University of Wisconsin Milwaukee UWM Digital Commons

Theses and Dissertations

May 2014

\title{
Nonconvex Cases for Carpenter's Rulers
}

\author{
Ke Chen \\ University of Wisconsin-Milwaukee
}

Follow this and additional works at: https://dc.uwm.edu/etd

Part of the Computer Sciences Commons

\section{Recommended Citation}

Chen, Ke, "Nonconvex Cases for Carpenter's Rulers" (2014). Theses and Dissertations. 577.

https://dc.uwm.edu/etd/577

This Thesis is brought to you for free and open access by UWM Digital Commons. It has been accepted for inclusion in Theses and Dissertations by an authorized administrator of UWM Digital Commons. For more information, please contact open-access@uwm.edu. 


\title{
Nonconvex Cases for Carpenter's Rulers
}

\author{
by \\ Ke Chen \\ A Thesis Submitted in \\ Partial Fulfillment of the \\ Requirements for the Degree of \\ Master of Science \\ in Computer Science
}

at

The University of Wisconsin-Milwaukee

May 2014 


\section{ABSTRACT \\ Nonconvex Cases for Carpenter's Rulers}

by

\section{Ke Chen}

The University of Wisconsin-Milwaukee, 2014

Under the Supervision of Professor Adrian Dumitrescu

We consider the carpenter's ruler folding problem in the plane, i.e., finding a minimum area shape with diameter 1 that accommodates foldings of any ruler whose longest link has length 1. An upper bound of 0.614 and a lower bound of 0.476 are known for convex cases. We generalize the problem to simple nonconvex cases: in this setting we improve the upper bound to 0.583 and establish the first lower bound of 0.073 . A variation is to consider rulers with at most $k$ links. The current best convex upper bounds are 0.486 for $k=3,4$ and 0.523 for $k=5,6$. These bounds also apply to nonconvex cases. We derive a better nonconvex upper bound of 0.296 for $k=3,4$. 
(C) Copyright by Ke Chen, 2014

All Rights Reserved 


\section{TABLE OF CONTENTS}

LIST OF FIGURES V v

LIST OF TABLES viii

1 Introduction 1

2 Upper Bound $\quad 8$

2.1 Diameter of $C \ldots \ldots \ldots \ldots \ldots$

2.2 Algorithm for folding a ruler inside $C \ldots \ldots$. . . . . . . . . . 10

2.3 Minimum area of $C \ldots \ldots \ldots \ldots$

3 Lower Bound $\quad 11$

3.1 Lower Bound with One 3-Link ruler . . . . . . . . . . . . . . . 11

3.2 Lower Bound with One 5-link Ruler . . . . . . . . . . . . . . . . . . 14

$4 k$-universal Cases $\quad 20$

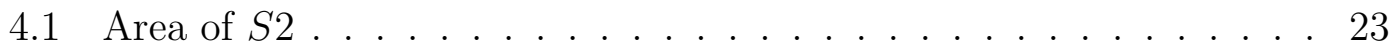

4.2 Area of $C 2 \ldots \ldots \ldots \ldots \ldots$

$4.3 C 2$ is 4 -universal . . . . . . . . . . . . . . . 26

5 Summary and Future Directions $\quad 29$

6 References $\quad 32$ 


\section{LIST OF FIGURES}

1 A universal case (in bold lines) for Moser's worm problem Left: a closed semidisk of unit diameter. Right: a plane curve of length 1 contained in the case. . . . . . . . . . . . . . . .

2 Another universal case (in bold lines) for Moser's worm problem. Left: a rhombus with a long diagonal of unit length and vertex angles of $60^{\circ}$ and $120^{\circ}$. Right: a plane curve of length 1 contained in the case.

3 A universal case (in bold lines) for Lebesgue's covering problem. Left: union of a unit Reuleaux triangle and a unit circle. Right: a Reuleaux triangle with unit diameter (shaded area) contained in the case. . . . . . . . . . . . . . . . . . .

4 Another universal case (in bold lines) for Lebesgue's covering problem. Left: a regular hexagon circumscribed to a unit circle, with two corners cut off. Right: a Reuleaux triangle with unit diameter (shaded area) contained in the case. . . . . . . . . . . . . . 3

5 A universal case (in bold lines) for Lebesgue's covering problem under translation. Left: a unit square. Right: a Reuleaux triangle with unit diameter (shaded area) can rotate inside the square. . . . 3

$6 \quad$ Smallest area translative universal case (in bold lines) for all open curves of unit length. Left: an equilateral triangle of unit height. Right: a unit open curve can rotate inside the equilateral triangle. .

7 The minimum folding length of a ruler can be almost double the length of its longest link. . . . . . . . . . . . . . . 4

8 Left: convex universal case $R 2$ (in bold lines). Right: folding a 6-link unit ruler $p_{0} p_{1} p_{2} p_{3} p_{4} p_{5} p_{6}$ into $R 2$.

9 Universal cases (in bold lines) are contained in a lens of radius 1. Left: convex universal case $R 2$. Right: nonconvex universal case $C$ for some $x \in[0,0.5] \ldots \ldots \ldots \ldots \ldots \ldots$

10 A nonconvex case (in bold lines) with a folded ruler inside. . . . . . 7

11 Left: nonconvex universal case $C$ for some $x \in[0,0.5]$; the shaded trapezoid can be discarded. Right: folding a 6-link unit ruler $p_{0} p_{1} p_{2} p_{3} p_{4} p_{5} p_{6}$ into $C$ 
12 Nonconvex universal case $C$ (in bold lines). . . . . . . . . . . . . 9

13 A plot of area $(C)$ (left) and its derivative (right). . . . . . . . 11

14 For a 3 -link ruler $1, t, 1$, where $t$ is fixed, the area of the shaded triangle is minimized when $\left|p_{0} p_{3}\right|=\left|p_{1} p_{3}\right|=1 \ldots \ldots . . \ldots 12$

15 Legend for the 5-link ruler used (in bold lines). . . . . . . . . . . . 15

16 Case 1 (left) and Case 2 (right): the lower bounds are given by the shaded areas in each case. . . . . . . . . . . . . . . . 16

17 Case 3: area above (left) and area below (right). The lower bound is given by the sum of the two shaded areas. . . . . . . . . . . 17

18 Case 4: area above (left) and area below (right). The lower bound is given by the sum of the two shaded areas. . . . . . . . . . . . . . 19

19 Left: 6-universal case $R 1$ (in bold lines). Right: folding a 6-link unit ruler $p_{0} p_{1} p_{2} p_{3} p_{4} p_{5} p_{6}$ into $R 1$. . . . . . . . . . . . . . . 21

20 Left: 4-universal case $R 1 / 2$ (in bold lines). Right: folding a 4-link unit ruler $p_{0} p_{1} p_{2} p_{3} p_{4}$ into $R 1 / 2 \ldots \ldots \ldots \ldots$. . . . . . . . 21

21 Left: convex 4-universal case $S 2$ (in bold lines). Right: folding a 4-link unit ruler $p_{0} p_{1} p_{2} p_{3} p_{4}$ into $S 2 \ldots \ldots \ldots \ldots . \ldots . \ldots . \ldots . \ldots$

22 Left: nonconvex 4-universal case $C 2$ (in bold lines). Right: folding a 4 -link unit ruler $p_{0} p_{1} p_{2} p_{3} p_{4}$ into $C 2 \ldots \ldots \ldots$. . . . . . 22

23 A plot of area $(S 2)$ (left) and its derivative (right). . . . . . . . 23

24 Derive $C 2$ from $S 2$. Left: $S 2$ (in bold lines) with the shaded triangle discarded. Right: $C 2$ (in bold lines) has minimum area $0.295 \ldots$ when $c$ lies in the middle of the circular arc $b d . \ldots . . . . . .24$

25 A plot of area $(C 2)$ (left) and its derivative (right) . . . . . . . 26

26 Two cases of folding a 3-link ruler into $C 2$ (in bold lines). Left: the middle link is short. Right: the middle link is long. . . . . . . . . . 26

27 Fold a 3-link ruler (in bold lines) into $C 2$. Left: $t \leq x$. Right: $t>x$. 27

28 Two cases of folding a 4-link ruler into $C 2$ (in bold lines). Left:

$p_{2} p_{3}$ is short. Right: $p_{2} p_{3}$ is long. . . . . . . . . . . . 27 
29 Fold a 4-link ruler (in bold lines) into $C 2$. Left: $\left|p_{2} c\right| \leq x$. Right: $\left|p_{2} c\right|>x \ldots \ldots \ldots \ldots \ldots \ldots$ 


\section{LIST OF TABLES}

1 Known bounds for convex cases. . . . . . . . . . . . . . . 7

2 Known and new (in bold) bounds for nonconvex cases. . . . . . . . 7 


\section{Introduction}

A universal case for a family of sets is a set that contains a copy (under congruence or translation) of each set in the family. Geometric questions on finding minimum universal cases for different families of sets have been studied for over a hundred years. Various measures of minimality such as smallest area and shortest diameter were also considered.

L. Moser's worm problem is one of the most famous problems of this type. It asks for a set in the plane with minimum area that can be used to cover any curve of length one. Meir showed that a closed semidisk of unit diameter (depicted in Figure 1 (left)) is a universal case for all curves of unit length. It has area $\pi / 8<0.3927$.
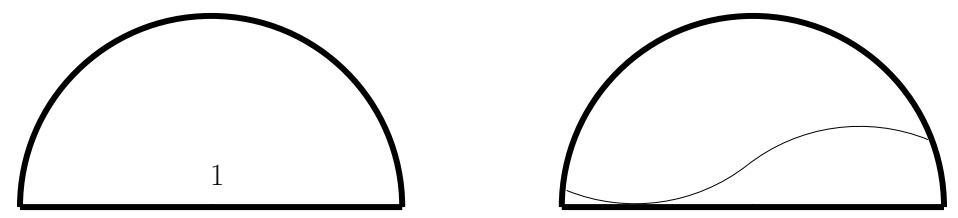

Figure 1: A universal case (in bold lines) for Moser's worm problem Left: a closed semidisk of unit diameter. Right: a plane curve of length 1 contained in the case.

Poole and Gerriets [6] constructed a smaller universal case from a rhombus (see Figure 2 (left)) whose long diagonal is of unit length and the larger angles are $120^{\circ}$. It has area $1 /(2 \sqrt{3})<0.2887$.
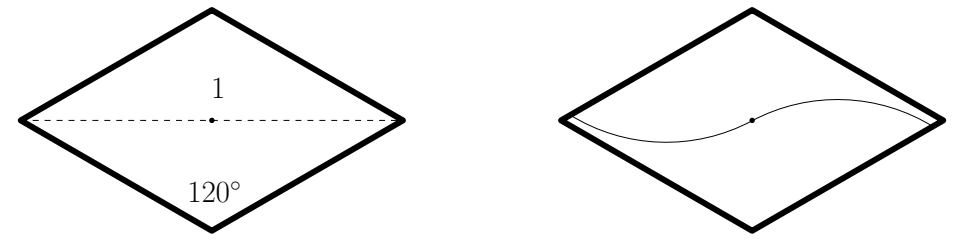

Figure 2: Another universal case (in bold lines) for Moser's worm problem. Left: a rhombus with a long diagonal of unit length and vertex angles of $60^{\circ}$ and $120^{\circ}$. Right: a plane curve of length 1 contained in the case.

Better upper bounds were gradually obtained over two decades since 1970s (see $[11,14,15])$. The current best upper bound for convex cases is 0.276 , derived 
by Norwood and Poole [10]. For nonconvex cases, a better upper bound of 0.246 was achieved by Hansen [7]. The current best convex lower bound 0.2194 is due to Wetzel [15] and dates back to 1973.

One of the oldest problems concerning universal cases was posed by Lebesgue. The problem asks for a smallest area convex universal case that contains a congruent copy of every planar set of unit diameter. A minimal universal case depicted in Figure 3 (left) is due to Eggleston [5]. It is the union of a Reuleaux triangle with diameter one and a unit circle where a pair of the triangle vertices is a diameter of the circle. It has area $1.0046 \ldots$.
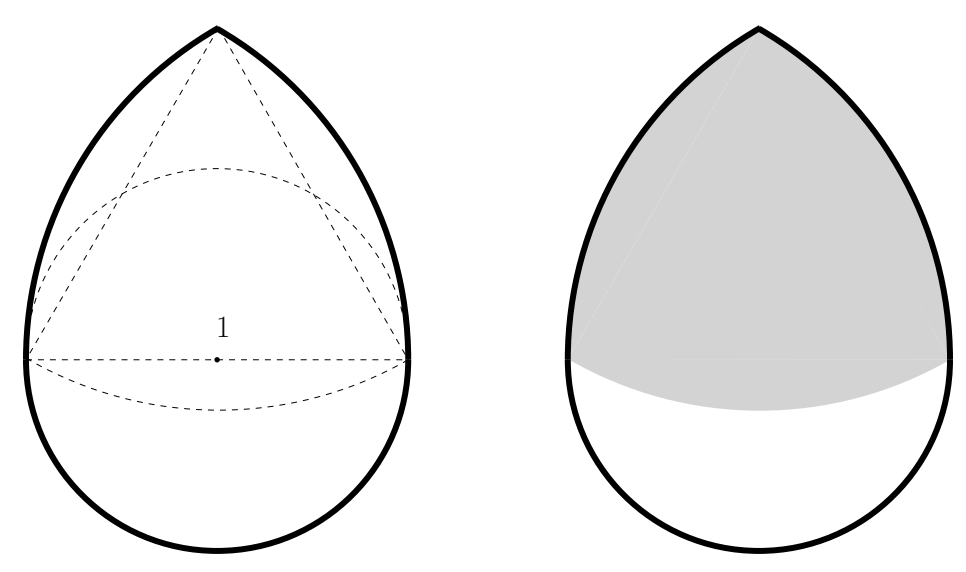

Figure 3: A universal case (in bold lines) for Lebesgue's covering problem. Left: union of a unit Reuleaux triangle and a unit circle. Right: a Reuleaux triangle with unit diameter (shaded area) contained in the case.

Pál [12] (see also [2, Problem 1, p. 457]) proved an upper bound of 0.8454 with his truncated hexagon (shown in Figure 4 (left)), a regular hexagon circumscribed to a unit circle, with two corners cut off. Pál also derived a lower bound of 0.8257 which was further increased to 0.832 by Braß and Sharifi [3].

Similar problems in which translation (but no rotation) is allowed were also studied. For the same problem, a universal case under translation is also a universal case under congruence. The direct analogue of Lebesgue's problem asks for a smallest universal case that contains a translate of every planar set of unit diameter. The unit square, shown in Figure 5 (left), is known to be a universal 

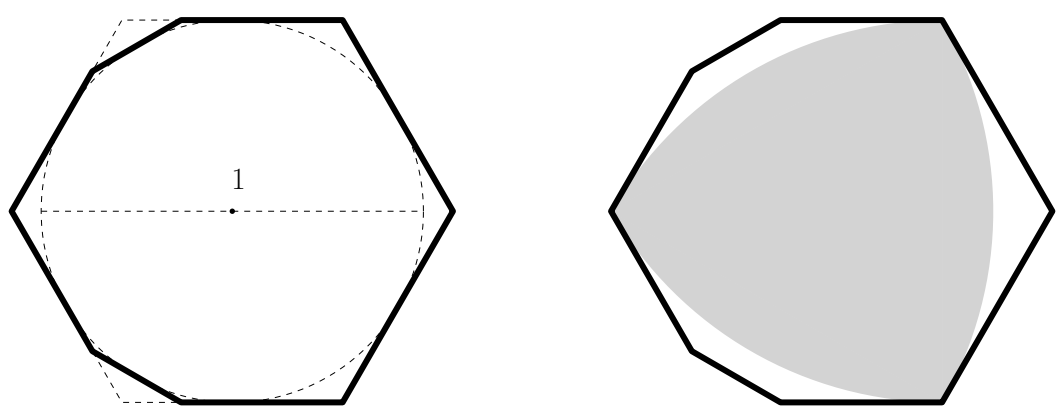

Figure 4: Another universal case (in bold lines) for Lebesgue's covering problem. Left: a regular hexagon circumscribed to a unit circle, with two corners cut off. Right: a Reuleaux triangle with unit diameter (shaded area) contained in the case.

case under translation (and congruence). One of the oldest results for universal cases under translation is due to Pál [13] who proved that the smallest universal translative case for all open curves of unit length is an equilateral triangle with height one and area $1 / \sqrt{3}$ (depicted in Figure 6 (left).
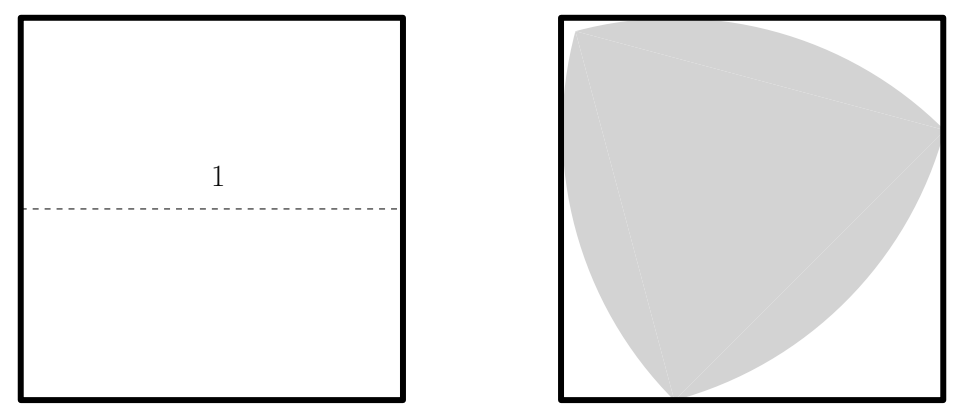

Figure 5: A universal case (in bold lines) for Lebesgue's covering problem under translation. Left: a unit square. Right: a Reuleaux triangle with unit diameter (shaded area) can rotate inside the square.

Another problem of finding universal (one dimensional) cases asks to determine the shortest interval into which a chain of line segments can be folded. A chain of line segments is called a carpenter's ruler consists of links of different lengths that are hinged together making it possible to fold. The problem was posed by Hopcroft, Joseph and Whitesides in [8] where the authors proved it to be NPcomplete and provided a factor 2-approximation algorithm. In [4], Călinescu and Dumitrescu improved this result by showing that there exists a fully polynomial- 

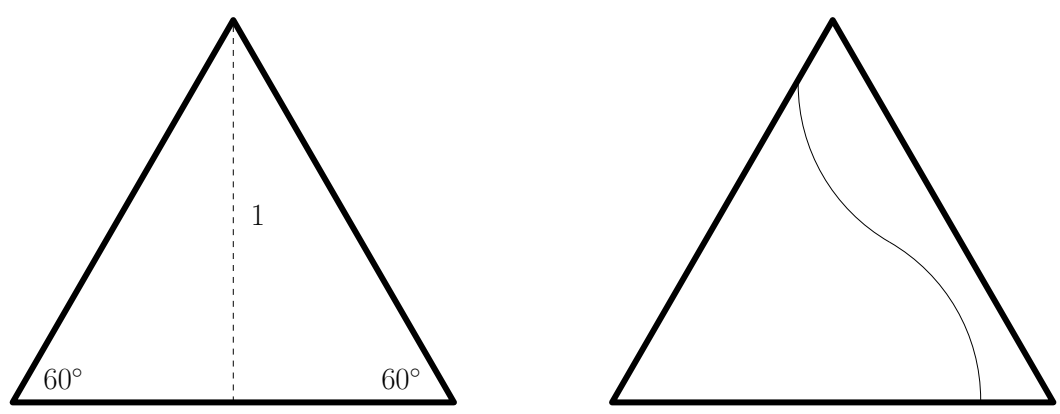

Figure 6: Smallest area translative universal case (in bold lines) for all open curves of unit length. Left: an equilateral triangle of unit height. Right: a unit open curve can rotate inside the equilateral triangle.

time approximation scheme. Hopcroft et al. [8] also observed that for a ruler with its longest link having length 1 , the minimum folding length can be at least $2-\epsilon$ for all $1 \geq \epsilon>0$. Consider a ruler with $2 n-1$ links as depicted in Figure 7, its links have lengths 1 and $1-\epsilon$ alternatively in which $n=\lceil 1 / \epsilon\rceil$. The minimum folding length of this ruler is $(n-1) \epsilon+1 \geq 2-\epsilon$.

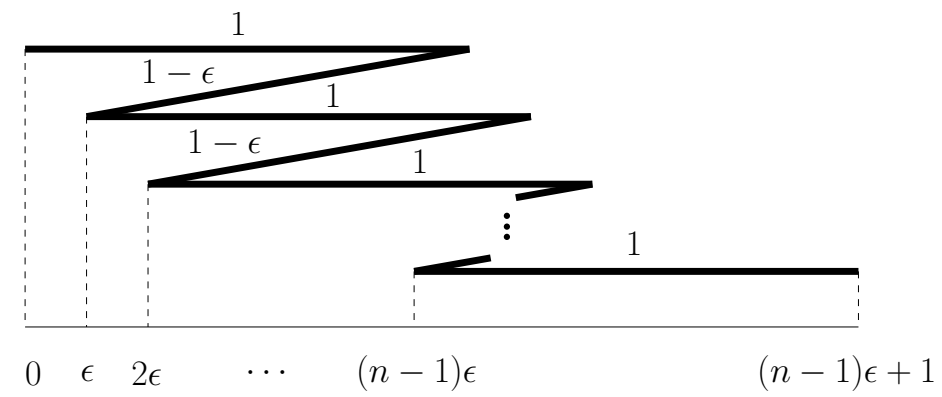

Figure 7: The minimum folding length of a ruler can be almost double the length of its longest link.

Călinescu and Dumitrescu [4] (see also [2, Problem 9, p. 461]) introduced a twodimensional version of the carpenter's ruler folding problem in 2005: finding the minimum area universal case for carpenter's rulers in the plane whose diameter is of the same length as a ruler's longest link. More formally, a carpenter's ruler $L$ of $n$ links is a chain of $n$ line segments with endpoints $p_{0}, p_{1}, \ldots, p_{n}$, with consecutive segments connected by hinges. For $0 \leq i \leq n-1$, the segment $p_{i} p_{i+1}$ is a link of the ruler. A ruler with its longest link having length 1 is called a unit ruler. A folding of a ruler $L$ is represented by the $n-2$ angles $\angle p_{i} p_{i+1} p_{i+2} \in[0, \pi]$ for all 
$0 \leq i \leq n-2$. A case is a planar shape whose boundary is a simple closed curve (i.e., with no self-intersections). In particular, a case has no interior holes.

Obviously a unit ruler requires a case whose diameter is at least one; on the other hand, there exist cases of unit diameter that allow folding of any unit ruler inside, e.g., a disk of unit diameter, regardless of the number of links in the ruler. A ruler $L$ can be folded inside a case $S$ if and only if there exists a point $p \in S$ and a folding of $L$ such that all the points on $L$ are in $S$ when $p_{0}$ is placed at $p$. In a folded position of the ruler, its links may cross each other; an example is shown in Figure 8 (right).

A case is said to be universal if any unit ruler (or all unit rulers) can be folded inside it. The question asks for the minimum area of a convex universal case of unit diameter. A disk of unit diameter and the Reuleaux triangle with one arc removed (call it $R 2$ ), were shown to be universal by the authors [4]. $R 2$ is depicted in Figure 8, its area is $\frac{\pi}{3}-\frac{\sqrt{3}}{4}=0.614 \ldots$; it is the current best upper bound for the area of a convex universal case. For any $n$-link unit ruler $p_{0}, p_{1}, \ldots, p_{n}$, a folding of it inside $R 2$ such that all $p_{i}$ 's lie on the circular arcs can be computed in $O(n)$ time. The authors [4] also achieved a lower bound of 0.375 using 3-link rulers, and this was further improved by Klein and Lenz [9] to 0.476 using 5-link rulers.
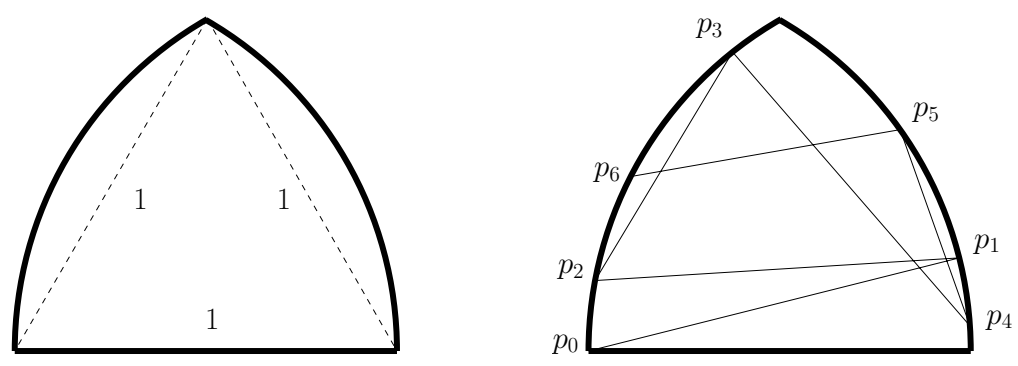

Figure 8: Left: convex universal case $R 2$ (in bold lines). Right: folding a 6-link unit ruler $p_{0} p_{1} p_{2} p_{3} p_{4} p_{5} p_{6}$ into $R 2$.

A case is $k$-universal if any unit ruler with at most $k$ links can be folded into it. In the problem of finding a universal case with minimum area, the number of links (as well as the total length) of the rulers is irrelevant. However, it is worth 
study if fewer links in the rulers allow better bounds. In [1], Alt et al. studied convex universal cases for rulers with a small number of links for which better upper bounds were achieved.

Since a universal case has unit diameter, it must be contained in a lens of radius 1 , namely the intersection of two disks of unit radius passing through the centers of each other (see Figure 9). It was shown in [9] that no subset of $R 2$ with a smaller area is universal. All previous work has focused on convex cases; the lower bounds were derived using convex hull of the rulers used in the respective arguments.
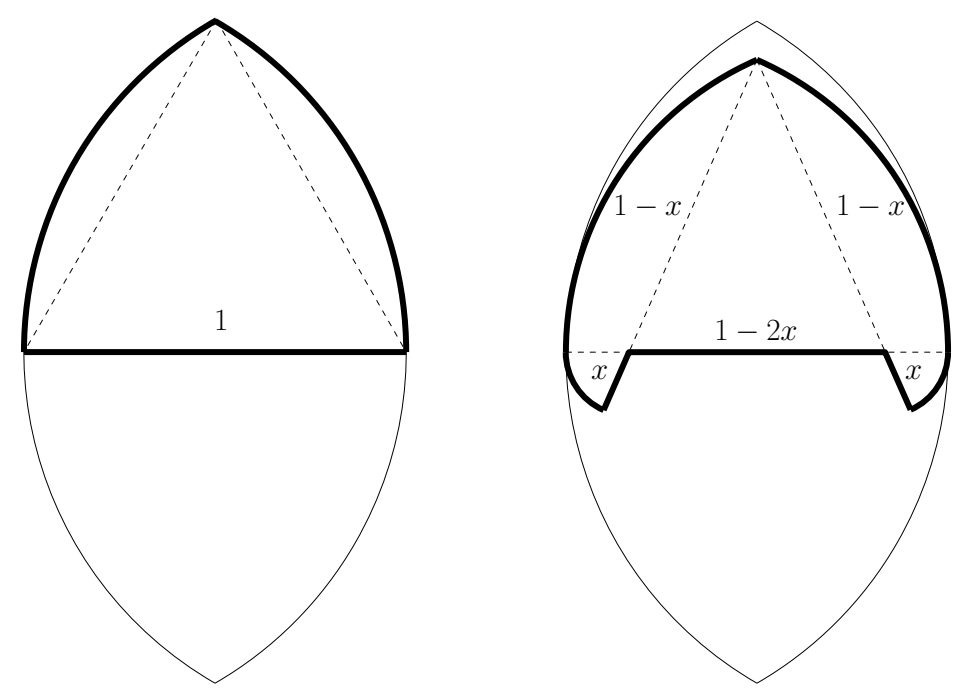

Figure 9: Universal cases (in bold lines) are contained in a lens of radius 1. Left: convex universal case $R 2$. Right: nonconvex universal case $C$ for some $x \in[0,0.5]$.

Călinescu and Dumitrescu [4] also asked whether the convexity of the case makes any difference. Here we deal with nonconvex cases, i.e., cases with spikes are allowed (see Figure 10), and give a first partial answer to this question. Our main result concerning nonconvex universal cases is summarized in the following theorem.

Theorem 1. There exists a (simple) nonconvex universal case $C$ of unit diameter and area at most 0.583. The folding of any unit ruler with $n$ links inside $C$ can 
be computed in $O(n)$ time. On the other hand, the area of any simple nonconvex universal case of unit diameter must be at least 0.073 .

For the problem of finding $k$-universal cases, our main result for $k=4$ is summarized in the following theorem.

Theorem 2. There exists a (simple) nonconvex 4-univeral case C2 of unit diameter and area at most 0.296. The folding of any unit ruler with at most 4 links inside $C 2$ can be computed in $O(1)$ time.

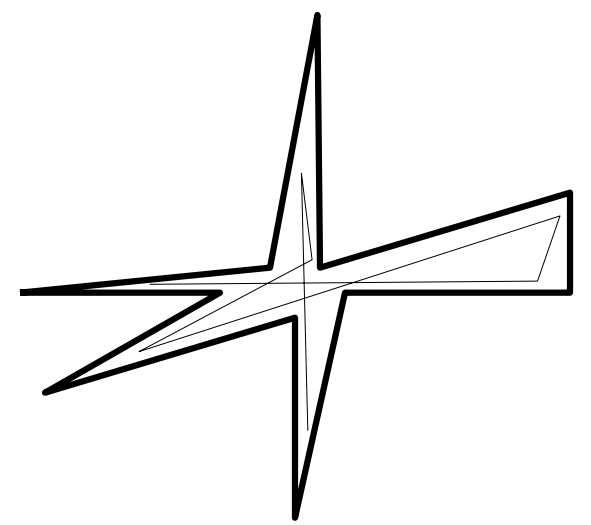

Figure 10: A nonconvex case (in bold lines) with a folded ruler inside.

Table 1 and Table 2 summarize the known and new bounds for convex and general (convex and nonconvex) cases respectively.

\begin{tabular}{|c|c|c|c|c|c|}
\hline & Universal & 3-universal & 4-universal & 5-universal & 6-universal \\
\hline Upper bounds & 0.614 & 0.486 & 0.486 & 0.523 & 0.523 \\
\hline Lower bounds & 0.476 & 0.375 & 0.375 & 0.476 & 0.476 \\
\hline
\end{tabular}

Table 1: Known bounds for convex cases.

\begin{tabular}{|c|c|c|c|c|c|}
\hline & Universal & 3-universal & 4-universal & 5-universal & 6-universal \\
\hline Upper bounds & $\mathbf{0 . 5 8 3}$ & $\mathbf{0 . 2 9 6}$ & $\mathbf{0 . 2 9 6}$ & 0.523 & 0.523 \\
\hline Lower bounds & $\mathbf{0 . 0 7 3}$ & $\mathbf{0 . 0 3 8}$ & $\mathbf{0 . 0 3 8}$ & $\mathbf{0 . 0 7 3}$ & $\mathbf{0 . 0 7 3}$ \\
\hline
\end{tabular}

Table 2: Known and new (in bold) bounds for nonconvex cases.

In Section 2, we prove that the nonconvex case $C$ shown in bold lines in Figure 11 is a universal case for any $x \in\left[0, \frac{1}{2}\right]$. Its area is at most 0.583 (achieved 

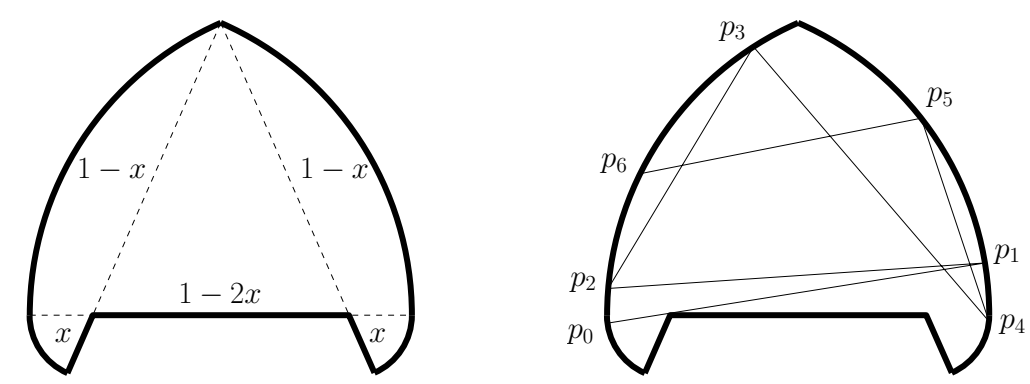

Figure 11: Left: nonconvex universal case $C$ for some $x \in[0,0.5]$; the shaded trapezoid can be discarded. Right: folding a 6 -link unit ruler $p_{0} p_{1} p_{2} p_{3} p_{4} p_{5} p_{6}$ into $C$.

when $x=0.165)$, i.e., smaller than the area of $R 2$. Notice that the case whose boundary is the convex hull of $C$ is a convex universal case whose area is at least 0.694 , larger than the area of $R 2$.

In Section 3, lower bounds for nonconvex universal cases are considered, i.e., only areas required by the simplicity of the case boundary are taken into account. We first derive a lower bound of 0.038 using a suitable 3-link ruler, and then extend the calculation to a suitable 5-link ruler and improve the lower bound to 0.073.

In Section 4 , the problem of finding $k$-universal cases for $k=4$ is considered. We construct another nonconvex case $C 2$ with unit diameter. It is proved to be 4-universal with an algorithm for folding unit rulers with at most 4 links inside it. $C 2$ has area at most 0.296 , smaller than 0.486 which is the current best upper bound for the area of a convex 4-universal case.

\section{Upper Bound}

The upper bound in Theorem 1 will be proved using the simple nonconvex shape $C$ shown in Figure 12. $C$ is constructed as follows.

- $|a c|=|a f|=|b g|=1$

- $|b d|=|c d|=|e f|=|e g|=x, x \in\left[0, \frac{1}{2}\right]$

- Arcs $a b$ and $g f$ are centered at $e$ with radii $1-x$ and $x$ respectively 
- Arcs $a g$ and $b c$ are centered at $d$ with radii $1-x$ and $x$ respectively

Notice that when $x=\frac{1}{2}, C$ becomes a disk with diameter 1 ; and when $x=0$, $C$ is identical to $R 2$. We show below that for any $x \in\left[0, \frac{1}{2}\right], C$ is a universal case with diameter 1 . Choosing $x=0.165$ yields a universal case with area $\leq 0.583$; notice that this area is smaller than $0.614 \ldots$, the area of $R 2$, the current smallest convex universal case.

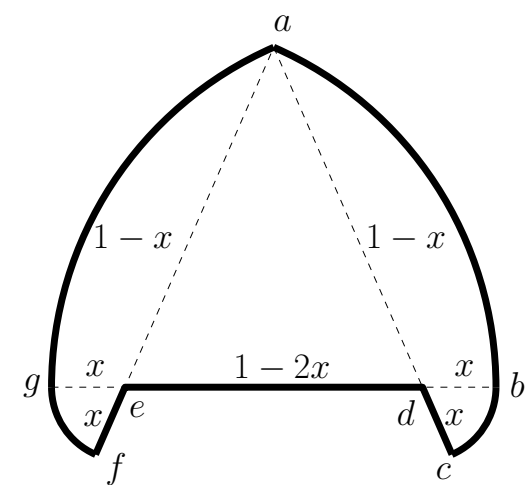

Figure 12: Nonconvex universal case $C$ (in bold lines).

\subsection{Diameter of $C$}

We show that $C$ has diameter 1 for any $x \in\left[0, \frac{1}{2}\right]$. The diameter is given by a pair of points on the convex hull, thus it suffices to consider points on $\operatorname{arcs} a b, b c, f g$, $g a$ and segment $c f$. Let $p$ and $p^{\prime}$ be two points on the convex hull of $C$.

Fix $p$ on arc $a b$. If $p^{\prime}$ is on $\operatorname{arc} a b,\left|p p^{\prime}\right| \leq|a b|<|a c|=1$. If $p^{\prime}$ is on arc $b c$ or segment $c f,\left|p p^{\prime}\right| \leq|a c|=1$. If $p^{\prime}$ is on arc $f g$, extend segment pe until it intersects arc $f g$ at point $p^{\prime \prime}$. If $p^{\prime}=p^{\prime \prime},\left|p p^{\prime}\right|=|p e|+\left|e p^{\prime \prime}\right|=1$; otherwise, segments $p e, e p^{\prime}, p p^{\prime}$ form a triangle, thus, $\left|p p^{\prime}\right|<|p e|+\left|e p^{\prime}\right|=|a e|+|e f|=1$. If $p^{\prime}$ is on $\operatorname{arc} g a,\left|p p^{\prime}\right| \leq|b g|=1$.

Fix $p$ on arc $b c$. If $p^{\prime}$ is on segment $c f,\left|p p^{\prime}\right| \leq|b f|<|b g|=1$. If $p^{\prime}$ is on arc $f g,\left|p p^{\prime}\right| \leq|b g|=1$. By symmetry, $C$ has diameter 1 . 


\subsection{Algorithm for folding a ruler inside $C$}

We show that the folding of any unit ruler with $n$ links inside $C$ can be computed in $O(n)$ time. We adapt the algorithm introduced in [4] to work with our case $C$. Fix the first free endpoint at some (arbitrary) point $p$ on a circular arc. Iteratively fix the next point of the ruler at some intersection point between the $\operatorname{arcs}$ of $C$ and the circle centered at $p$ with radius the length of the current link.

Notice that for any point $p$ on the circular arcs of $C$, and for any $t \in[0,1]$, there exists at least one point $p^{\prime}$ on these arcs such that $\left|p p^{\prime}\right|=t$. This guarantees the existence of the intersection points used in the iterative steps of the above algorithm.

\subsection{Minimum area of $C$}

The area of $C$ is the sum of areas of the sectors $d a g, d b c, e a b$ and $e f g$ minus the area of the triangle $\Delta a d e$. In the triangle $\Delta a d e$, we have $\angle a d e=\arccos \frac{1-2 x}{2-2 x}$. The sectors $d a g$ and $e a b$ have the same area $\frac{(1-x)^{2}}{2} \arccos \frac{1-2 x}{2-2 x}$. The sectors $d b c$ and efg have the same area $\frac{x^{2}}{2} \arccos \frac{1-2 x}{2-2 x}$.

The triangle $\Delta$ ade has area $\frac{1-2 x}{4} \sqrt{3-4 x}$. It follows that

$$
\begin{aligned}
\operatorname{area}(C) & =2 \operatorname{area}(\text { sector } d a g)+2 \operatorname{area}(\text { sector } d b c)-\operatorname{area}(\Delta a d e) \\
& =(1-x)^{2} \arccos \frac{1-2 x}{2-2 x}+x^{2} \arccos \frac{1-2 x}{2-2 x}-\frac{1-2 x}{4} \sqrt{3-4 x} \\
& =\left(1-2 x+2 x^{2}\right) \arccos \frac{1-2 x}{2-2 x}+\frac{2 x-1}{4} \sqrt{3-4 x} .
\end{aligned}
$$

Taking derivatives yields

$$
\frac{d(\operatorname{area}(C))}{d x}=(4 x-2) \arccos \frac{1-2 x}{2-2 x}+\frac{3-7 x+5 x^{2}}{(1-x) \sqrt{3-4 x}} .
$$



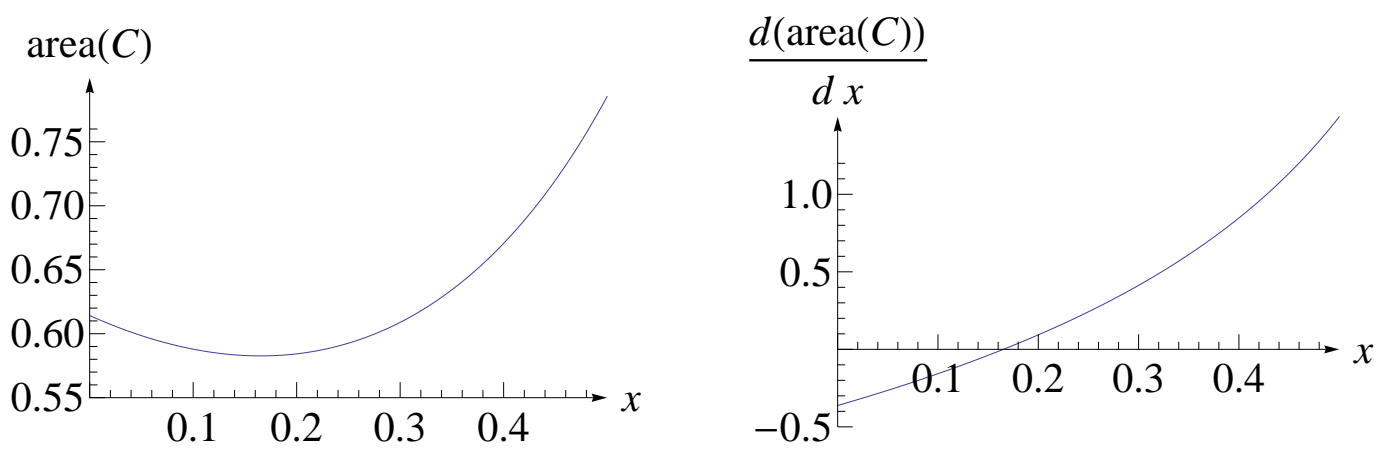

Figure 13: A plot of area $(C)$ (left) and its derivative (right).

Solving for $\frac{d(\operatorname{area}(C))}{d x}=0$ yields a single root $x=0.165 \ldots$, at which $C$ has the smallest area, area $(C) \leq 0.583$ (see Figure 13).

\section{Lower Bound}

We start with Lemma 1 (in Subsection 3.1), which gives a lower bound of 0.038 for the area required by a suitable 3-link ruler. As it turns out, this lower bound is the best possible for all 3-link rulers. Lemma 1 will be reused when deriving a lower bound for 5-link rulers (in Subsection 3.2), improving this first bound to 0.073 .

\subsection{Lower Bound with One 3-Link ruler}

For 3-link rulers, it is sufficient to consider the sequence of lengths $1, t, 1$ with $t \in(0,1)$. Indeed, given a folding of ruler $1, t, 1$, and an arbitrary unit 3-link ruler with links $a, t, b$, make the $t$-links of the two rulers coincide, and fold the $a$ - and $b$-links over the two unit links; the resulting folding is a valid one in the same case required by the $1, t, 1$ ruler.

For the 3-link ruler with link lengths 1,t,1, the two 1-links must intersect otherwise the diameter constraint will be violated, see Figure 14. The shaded triangle is the only area that counts for the nonconvex lower bound. 


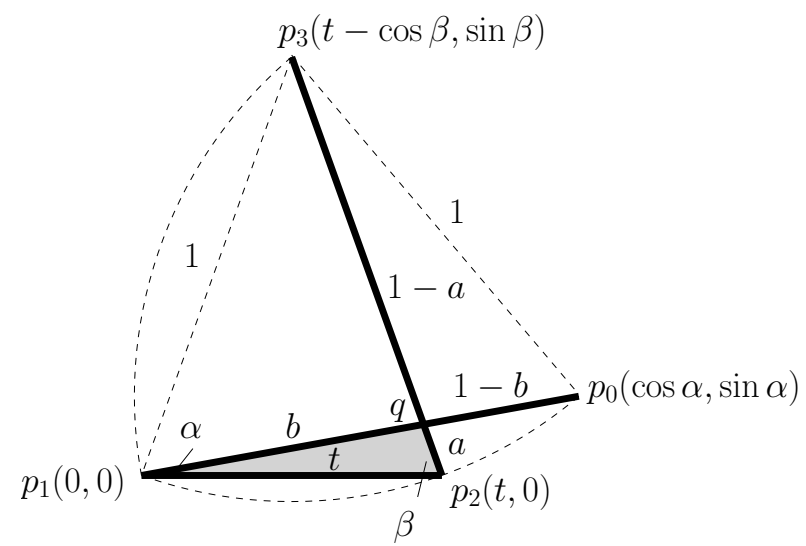

Figure 14: For a 3-link ruler 1,t,1, where $t$ is fixed, the area of the shaded triangle is minimized when $\left|p_{0} p_{3}\right|=\left|p_{1} p_{3}\right|=1$.

Lemma 1. For any $t \in(0,1)$, the shaded triangle in Figure 14 is minimized when $\alpha=\arccos \frac{t}{2}-\frac{\pi}{3}$ and $\beta=\arccos \frac{t}{2}$.

Proof. By symmetry, we can assume that $\alpha \leq \beta$. Denote the area of the shaded triangle $\Delta p_{1} p_{2} q$ by $S$. Since the triangle has base $t$, its height $h$ determines the area. The height $h$ is the distance between $p_{1} p_{2}$ and the intersection point between $p_{0} p_{1}$ and $p_{2} p_{3}$. For any fixed $\alpha \in\left[\arccos \frac{t}{2}-\frac{\pi}{3}, \arccos \frac{t}{2}\right]$, the area is minimized when $\beta$ is minimized without violating the diameter constraint $\left|p_{0} p_{3}\right| \leq 1$. Denote this angle by $\beta(\alpha) ; \beta(\alpha)$ is a monotonically decreasing function that can be determined by computing the intersection of two circles of radius 1 centered at $p_{0}$ and $p_{2}$. In the following discussion, we will refer to this angle by $\beta$.

It suffices to express the area $S$ as a function of two parameters, $t$ and $\alpha$. In fact, $h \cot \alpha+h \cot \beta=t$ or

$$
h=\frac{t}{\cot \alpha+\cot \beta} .
$$

So

$$
S(t, \alpha)=\frac{t h}{2}=\frac{t^{2}}{2(\cot \alpha+\cot \beta)} .
$$


Taking derivative with respect to $\alpha$, we have

$$
\frac{d S(t, \alpha)}{d \alpha}=\frac{t^{2}}{2(\cot \alpha+\cot \beta)^{2}}\left(\frac{1}{\sin ^{2} \alpha}+\frac{1}{\sin ^{2} \beta} \frac{d \beta}{d \alpha}\right)
$$

To see that $S$ is minimized when $\alpha$ is minimized, we need to show that $\frac{d S(t, \alpha)}{d \alpha}>0$, i.e.,

$$
\begin{gathered}
\frac{1}{\sin ^{2} \alpha}+\frac{1}{\sin ^{2} \beta} \frac{d \beta}{d \alpha}>0, \text { or } \\
\frac{d \beta}{d \alpha}>-\frac{b^{2}}{a^{2}} .
\end{gathered}
$$

Suppose $p_{1}$ is fixed at $(0,0), p_{2}$ is at $(t, 0)$, then $p_{0}$ has coordinates $(\cos \alpha, \sin \alpha)$, $p_{3}$ has coordinates $(t-\cos \beta, \sin \beta)$. Since $\left|p_{0} p_{3}\right|=1$, we have

$$
\begin{aligned}
& (t-\cos \beta-\cos \alpha)^{2}+(\sin \beta-\sin \alpha)^{2}=1, \text { or } \\
& t^{2}+1-2 t \cos \beta-2 t \cos \alpha+2 \cos (\alpha+\beta)=0 .
\end{aligned}
$$

Taking derivative with respect to $\alpha$, we have

$$
\begin{gathered}
2 t \sin \beta \frac{d \beta}{d \alpha}+2 t \sin \alpha-2 \sin (\alpha+\beta)\left(1+\frac{d \beta}{d \alpha}\right)=0, \text { or } \\
\frac{d \beta}{d \alpha}=\frac{\sin (\alpha+\beta)-t \sin \alpha}{t \sin \beta-\sin (\alpha+\beta)} .
\end{gathered}
$$

Notice that in the shaded triangle $\Delta p_{1} p_{2} q$,

$$
\frac{a}{\sin \alpha}=\frac{b}{\sin \beta}=\frac{t}{\sin (\alpha+\beta)} .
$$

So

$$
\frac{d \beta}{d \alpha}=-\frac{1-a}{1-b}
$$


Thus $\frac{d S(t, \alpha)}{d \alpha}>0$ is equivalent to

$$
\begin{gathered}
\frac{d \beta}{d \alpha}=-\frac{1-a}{1-b}>-\frac{b^{2}}{a^{2}}, \text { or } \\
b^{2}-b^{3}>a^{2}-a^{3} .
\end{gathered}
$$

This inequality holds if $0<a<2 / 3$ and $a<b<(1-a) / 2+\sqrt{(1+3 a)(1-a)} / 2$. We know this is true since in triangle $\Delta p_{0} p_{3} q,(1-a)+(1-b)>1$. Recall $\alpha \leq \beta$, so $a \leq b$. If $a>1 / 2,(1-a)+(1-b) \leq 2(1-a)<1$, so $a \leq 1 / 2$. And $b<1-a<(1-a) / 2+\sqrt{(1-a)^{2}} / 2$.

Observe that $\alpha$ and the correspondingly $\beta$ are determined when $\left|p_{1} p_{3}\right|=1$ (see Figure 14). Moreover, this value of $\alpha$ is the minimum possible; indeed, if $\alpha$ is getting smaller, either $\left|p_{0} p_{3}\right|$ or $\left|p_{1} p_{3}\right|$ will violate the diameter constraint. In the isosceles triangle $\Delta p_{1} p_{2} p_{3}$, we have $\beta=\alpha+\angle p_{0} p_{1} p_{3}$ and $\cos \beta=\frac{t}{2}$. In the equilateral triangle $\Delta p_{0} p_{1} p_{3}$, we have $\angle p_{0} p_{1} p_{3}=60^{\circ}$. So $\beta=\alpha+\frac{\pi}{3}=\arccos \frac{t}{2}$.

Now we are ready to show our first lower bound on simple nonconvex cases. By Lemma 1,

$$
S(t, \alpha) \geq U(t):=\frac{t^{2}}{2\left(\cot \left(\arccos \frac{t}{2}-\frac{\pi}{3}\right)+\cot \arccos \frac{t}{2}\right)}
$$

It is easy to check that $U(0.676) \geq 0.038$, as desired. For $t \in(0,1), U(t)$ attains its maximum value for $t=0.676 \ldots$, and this is the best possible bound for a single 3-link ruler.

\subsection{Lower Bound with One 5-link Ruler}

Consider a special ruler with 5 links of lengths 1, 0.6, 1, 0.6, 1 as shown in Figure 15. Recall that all the 1-links must pairwise intersect. Since the ruler is symmetric, w.l.o.g., we can assume that $\beta \geq \gamma$. The following lemma gives a 
better lower bound using this ruler.

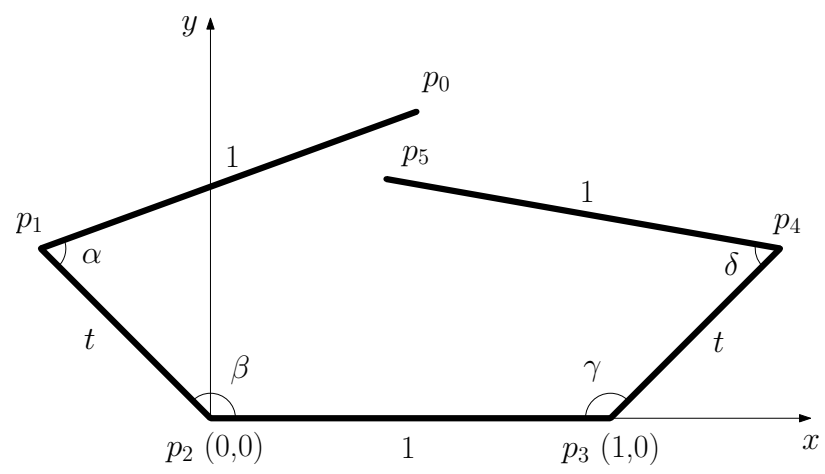

Figure 15: Legend for the 5-link ruler used (in bold lines).

Lemma 2. The minimum area of a simple (nonconvex) case of unit diameter required by folding the ruler 1, 0.6, 1, 0.6, 1 inside it is at least 0.073 .

Proof. Put $t=0.6$. The Cartesian coordinate is set up as follows: fix the origin at $p_{2}$ and let the $x$-axis pass through $p_{3}$. We have $p_{2}=(0,0), p_{3}=(1,0)$, $p_{1}=(t \cos \beta, t \sin \beta)$ and $p_{4}=(1-t \cos \gamma, t \sin \gamma)$. Recall that the case is required to be simple, i.e., no self-intersections or holes are allowed. According to the analysis of 3 -link rulers, $\beta, \gamma \in\left[\arccos \frac{t}{2}-\frac{\pi}{3}\right.$, $\left.\arccos \frac{t}{2}\right]$. We distinguish four cases according to the angles $\beta$ and $\gamma$.

Case 1: The two t-links do not intersect. This case includes the situation that $p_{3} p_{4}$ is folded below $p_{2} p_{3}$. As shown in Figure 16 (left), each shaded triangle is minimized using Lemma 1.

$$
\beta=\gamma=\arccos \frac{t}{2}=72.54 \ldots \circ, \quad \alpha=\delta=\arccos \frac{t}{2}-\frac{\pi}{3}=12.54 \ldots \circ
$$

Observe that this is not a valid folding since the two 1-links $p_{0} p_{1}$ and $p_{4} p_{5}$ do not intersect. However, it gives a valid lower bound since for any fixed $\beta$ and $\gamma$, increasing $\alpha$ or $\delta$ (to make the 1-links intersect) will increase the total area. By 
(1), the lower bound for Case 1 is

$$
2 U(t)=\frac{t^{2}}{\cot \left(\arccos \frac{t}{2}-\frac{\pi}{3}\right)+\cot \arccos \frac{t}{2}} \geq 0.074
$$
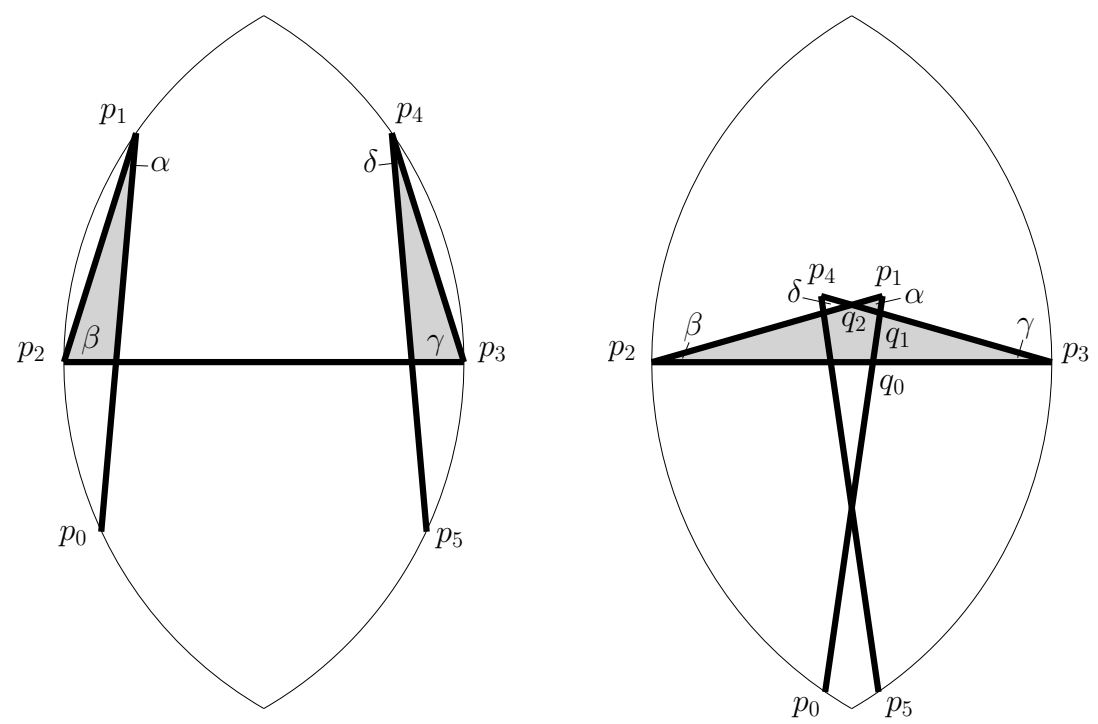

Figure 16: Case 1 (left) and Case 2 (right): the lower bounds are given by the shaded areas in each case.

Case 2: The two t-links intersect and both $\beta$ and $\gamma$ are at least $16^{\circ}$. As shown in Figure 16 (right), increasing $\beta$ or $\gamma$ will enlarge the upper shaded area consisting of the triangles $\Delta q_{0} p_{1} p_{2}$ and $\Delta q_{0} p_{3} q_{1}$. The area of the triangle below $p_{2} p_{3}$ will decrease but we simply ignore it when computing the lower bound in this case. Similar to the case of 3 -link rulers, when $\beta=\gamma=16^{\circ}, \alpha$ should be minimized under the constraint $\left|p_{0} p_{3}\right| \leq 1$ otherwise the area of the upper right small triangle $\Delta q_{1} p_{1} q_{2}$ will increase. In this configuration, triangle $\Delta q_{0} p_{1} p_{2}$ has height $t \sin \beta$. Its base $\left|p_{2} q_{0}\right|$ is the difference between the projections of the segments $p_{2} p_{1}$ and $q_{0} p_{1}$ on the $x$-axis, and $\angle p_{1} q_{0} p_{3}=\alpha+\beta$. It follows that

$$
b=\left|p_{2} q_{0}\right|=t \cos \beta-\frac{t \sin \beta}{\tan (\alpha+\beta)} .
$$


Triangle $\Delta q_{0} p_{3} q_{1}$ has base $1-b$. Its height $h$ equals to the $y$-coordinate of $q_{1}$ which is the intersection point of lines $p_{0} p_{1}$ and $p_{3} p_{4}$. The equation of line $p_{0} p_{1}$ is

$$
y=\tan (\alpha+\beta)(x-t \cos \beta)+t \sin \beta .
$$

The equation of line $p_{3} p_{4}$ is

$$
y=(1-x) \tan \gamma
$$

The $y$-coordinate of their intersection is

$$
h=\frac{(t \sin \beta+(1-t \cos \beta) \tan (\alpha+\beta)) \tan \gamma}{\tan \gamma+\tan (\alpha+\beta)} .
$$

The total shaded area is the sum of the two areas of triangles $\Delta q_{0} p_{1} p_{2}$ and $\Delta q_{0} p_{3} q_{1}$, namely

$$
\frac{b t \sin \beta+(1-b) h}{2} \geq 0.073
$$

Case 3: The two t-links intersect and $\beta \geq 16^{\circ}, \gamma \leq 16^{\circ}$. In this case, the lower
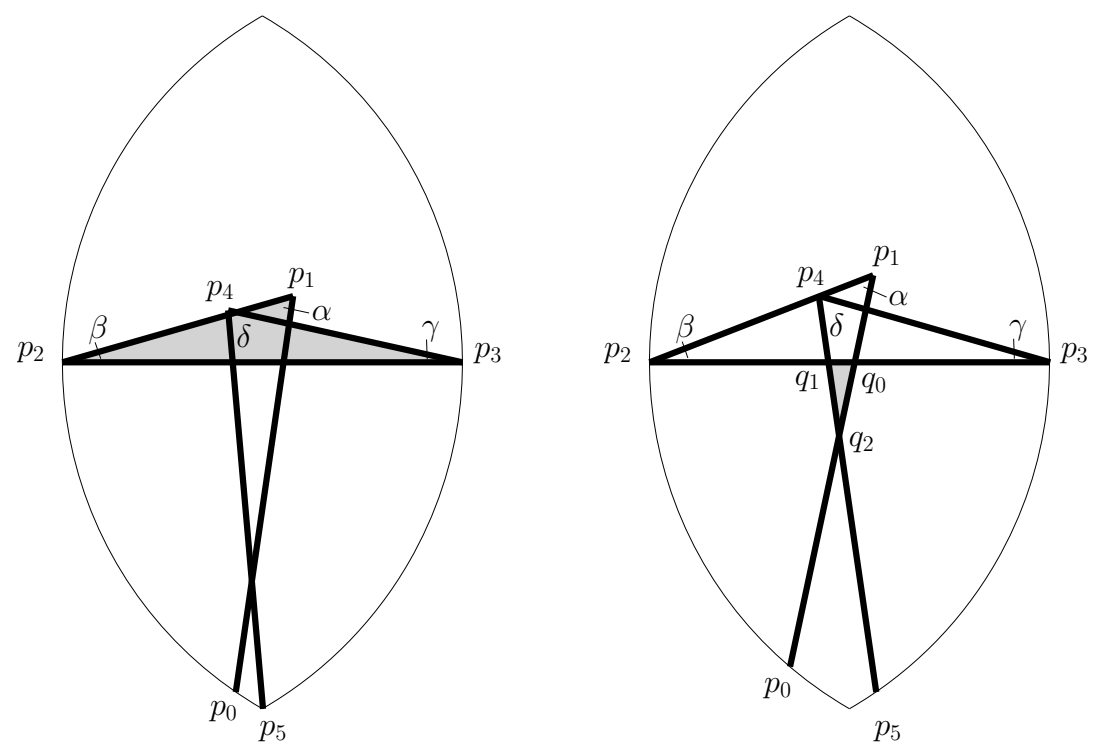

Figure 17: Case 3: area above (left) and area below (right). The lower bound is given by the sum of the two shaded areas. 
bound consists of two parts, the minimum shaded areas above and below $p_{2} p_{3}$, denoted by $S_{a}$ and $S_{b}$ respectively.

As shown in Figure 17 (left), with a similar argument as in Case 2, the minimum shaded area above $p_{2} p_{3}$ is achieved when $\beta=16^{\circ}, \gamma=\arccos \frac{t}{2}-\frac{\pi}{3}$ (which is the minimum value) and $\alpha$ is minimized under the constraint $\left|p_{0} p_{3}\right| \leq 1$. Plugging in these values into (2), (3) and (4) in Case 2 yields $S_{a} \geq 0.067$.

Observe that when $\beta$ and $\gamma$ increase, $\alpha$ and $\delta$ can take smaller values under the constraints $\left|p_{0} p_{3}\right| \leq 1,\left|p_{2} p_{5}\right| \leq 1$ and thus form a smaller triangle below $p_{2} p_{3}$. So the area of triangle $\Delta q_{0} q_{1} q_{2}$ is minimized when both $\beta$ and $\gamma$ take the maximum values, i.e., $\gamma=16^{\circ}$ and $\beta$ is chosen such that $p_{4}$ lies on $p_{1} p_{2}\left(p_{1} p_{2}\right.$ and $p_{3} p_{4}$ need to intersect). Then, both $\alpha$ and $\delta$ are minimized under the diameter constraints. This configuration is shown in Figure 17 (right). Similar to (2), we have

$$
\begin{aligned}
& \left|p_{2} q_{0}\right|=t \cos \beta-\frac{t \sin \beta}{\tan (\alpha+\beta)} \\
& \left|q_{1} p_{3}\right|=t \cos \gamma-\frac{t \sin \gamma}{\tan (\gamma+\delta)}
\end{aligned}
$$

The base of triangle $\Delta q_{0} q_{1} q_{2}$ is $b=\left|p_{2} q_{0}\right|+\left|q_{1} p_{3}\right|-1$. The height $h$ of this triangle is the absolute value of the $y$-coordinate of $q_{2}$, the intersection point of lines $p_{0} p_{1}$ and $p_{4} p_{5}$. The equation of line $p_{0} p_{1}$ is

$$
y=\tan (\alpha+\beta)(x-t \cos \beta)+t \sin \beta
$$

The equation of line $p_{4} p_{5}$ is

$$
y=\tan (\gamma+\delta)(1-t \cos \gamma-x)+t \sin \gamma
$$


Solving for their intersection point gives

$$
\begin{gathered}
h=\frac{\tan (\alpha+\beta) \tan (\gamma+\delta)(t \cos \beta+t \cos \gamma-1)}{\tan (\alpha+\beta)+\tan (\gamma+\delta)} \\
-\frac{t \tan (\gamma+\delta) \sin \beta+t \tan (\alpha+\beta) \sin \gamma}{\tan (\alpha+\beta)+\tan (\gamma+\delta)} .
\end{gathered}
$$

It follows that $S_{b}=\frac{1}{2} h b \geq 0.006$, and consequently, the minimum total shaded area is $S_{a}+S_{b} \geq 0.073$.

Case 4: both $\beta$ and $\gamma$ are no more than $16^{\circ}$. Notice that since $t=0.6$, the two $t$-links must intersect. Similar to Case 3, the lower bound is calculated as
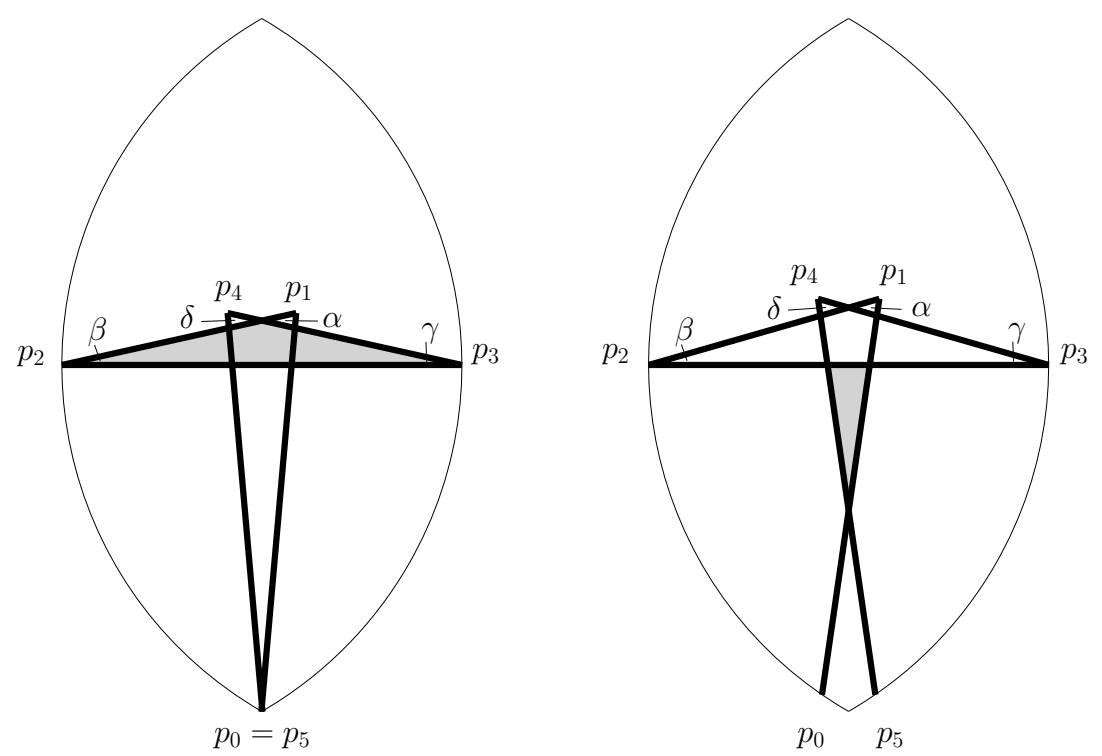

Figure 18: Case 4: area above (left) and area below (right). The lower bound is given by the sum of the two shaded areas.

the sum of minimized areas of shaded triangles above and below $p_{2} p_{3}$. For the triangle above $p_{2} p_{3}$, recall that $\beta$ and $\gamma$ both have the minimum possible value, $\arccos \frac{t}{2}-\frac{\pi}{3}$, as shown in Figure 18 (left). The minimized isosceles triangle above $p_{2} p_{3}$ has base 1 and height $\frac{\tan \beta}{2}$. Its area is

$$
S_{a}=\frac{\tan \left(\arccos \frac{t}{2}-\frac{\pi}{3}\right)}{4} \geq 0.055 .
$$


The area of the triangle below $p_{2} p_{3}$ is minimized when both $\beta$ and $\gamma$ take the maximum value $\left(16^{\circ}\right)$. Using (5) and (6) in Case 3 and $\alpha=\delta, \beta=\gamma$, the triangle below $p_{2} p_{3}$ has base

$$
b=2\left(t \cos \beta-\frac{t \sin \beta}{\tan (\alpha+\beta)}\right)-1
$$

and height

$$
h=\frac{(2 t \cos \beta-1) \tan (\alpha+\beta)}{2}-t \sin \beta .
$$

Its area is $S_{b}=\frac{h b}{2} \geq 0.019$. The minimum total shaded area is $S_{a}+S_{b} \geq 0.074$.

In summary, by Cases 2 and 3 of the analysis, the minimum nonconvex area required by folding the ruler $1,0.6,1,0.6,1$ within a case of unit diameter is at least 0.073 .

\section{$4 \quad k$-universal Cases}

In this section, we consider the problem of finding $k$-universal cases of minimum areas. Let $A_{k}$ be the smallest area of a convex $k$-universal case and $B_{k}$ be the smallest area of an arbitrary (convex or nonconvex) $k$-universal case. For any $k \in \mathbb{N}, B_{k} \leq A_{k}$. Additionally, $\forall i<j \in \mathbb{N}, A_{i} \leq A_{j}, B_{i} \leq B_{j}$. Our goal is to find better bounds for $B_{k}$. This problem was first studied by Alt et al. [1], in which the authors proved that $A_{3} \leq A_{4}<0.486$ and $A_{5} \leq A_{6} \leq 0.523$. With a simple nonconvex case $C 2$ (see Figure 22), we derive a better upper bound, $B_{4}<0.296$, which is smaller than the current best upper bound of $A_{4}<0.486$.

Replacing an circular arc in $R 2$ (Figure 8) with its chord results in the case $R 1$, depicted in Figure 19, a sector with radius one and center angle $60^{\circ}$, its area is $\pi / 6$. Alt et al. [1] proved that $R 1$ is 6 -universal but not 7 -universal. Thus, $A_{6} \leq \operatorname{area}($ sector $a b c)=\pi / 6=0.532 \ldots$.

Further replacing half of the remaining circular arc in $R 1$ with a line segment 

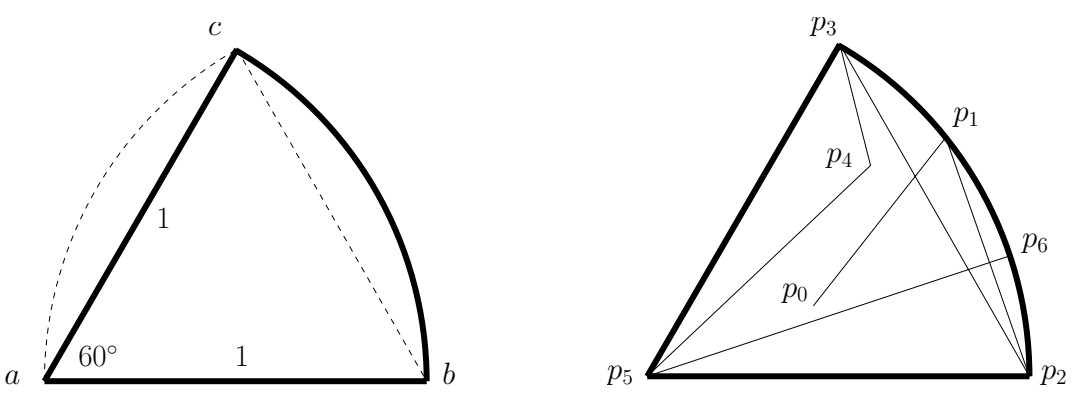

Figure 19: Left: 6-universal case $R 1$ (in bold lines). Right: folding a 6-link unit ruler $p_{0} p_{1} p_{2} p_{3} p_{4} p_{5} p_{6}$ into $R 1$.
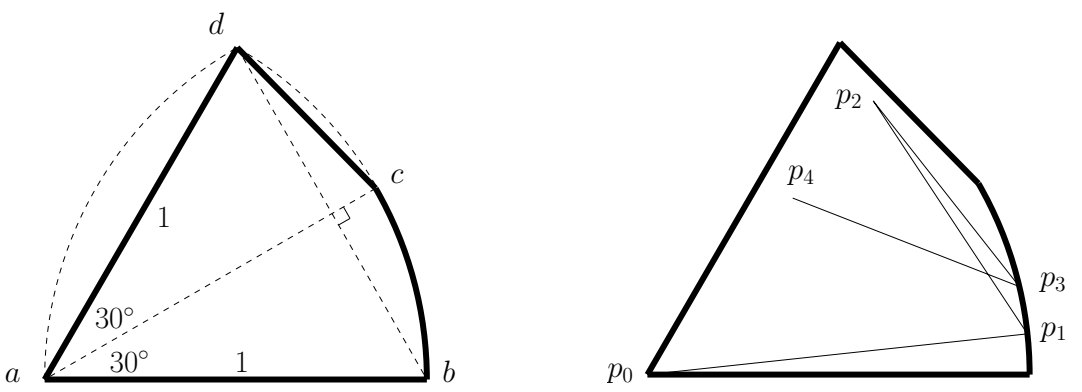

Figure 20: Left: 4-universal case $R 1 / 2$ (in bold lines). Right: folding a 4-link unit ruler $p_{0} p_{1} p_{2} p_{3} p_{4}$ into $R 1 / 2$.

produces the case $R 1 / 2$ shown in Figure 20. $R 1 / 2$ consists of the sector $a b c$ with radius one and center angle $30^{\circ}$ and the isosceles triangle $\Delta a c d$ with base $|a c|=1$ and height $|b d| / 2=1 / 2$. $R 1 / 2$ was shown to be 4 -universal but not 5 -universal in [1]. Thus, $A_{4} \leq \operatorname{area}($ sector $a b c)+\operatorname{area}(\Delta a c d)=\pi / 12+1 / 4=0.512 \ldots$
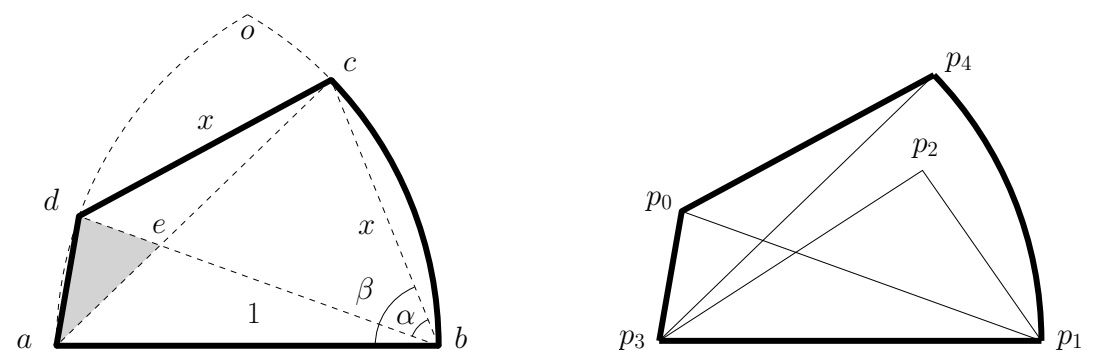

Figure 21: Left: convex 4-universal case $S 2$ (in bold lines). Right: folding a 4-link unit ruler $p_{0} p_{1} p_{2} p_{3} p_{4}$ into $S 2$.

Alt et al. [1] improved the upper bounds of $A_{4}$ with a better 4-universal case $S 2$ depicted in Figure 21. $S 2$ is constructed as follows. $|a b|=1$, pick an arbitrary 
point $c$ on the circular arc $o b$, let $x=|b c|$. Draw a circle centered at $c$ with radius $x$, let it intersect arc oa at point $d$. Notice that $d$ exists only if $|o c| \leq|c b|$. So $c$ must be at the middle point of arc $o b$ or higher, i.e., $\angle c a b \geq 30^{\circ}$. In $\Delta a b c$, $|b c|=x,|a c|=|a b|=1$. Thus $|b c|=|c d|=x \geq \sqrt{2-\sqrt{3}}=0.517 \ldots$ Similar to the universal case $C$ introduced in Section 2, $S 2$ is proved to be 4-universal with any value of $x \in[\sqrt{2-\sqrt{3}}, 1]$. Notice that when $x=1, S 2$ becomes the 6-universal case $R 1$; when $c$ lies at the middle of the arc $o b, S 2$ is identical to $R 1 / 2$.

The minimum area of $S 2$ was claimed (without proof) to be approximately 0.485. We confirm this result by providing full details in Section 4.1. However, we observe that the shaded triangle $\Delta a d e$ in Figure 21 (left) is not necessary for the folding algorithm introduced in [1]. If we discard this triangle, a family of nonconvex cases abcde with parameter $x$ can be obtained. In Section 4.2, we
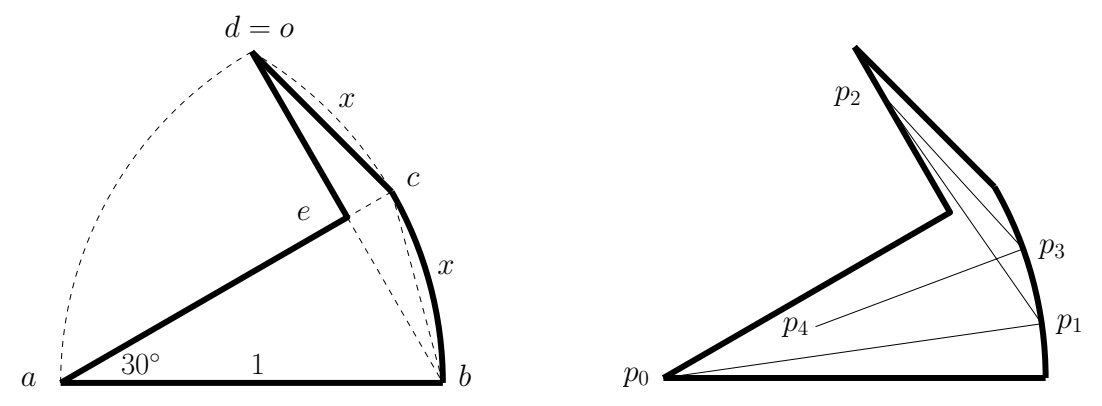

Figure 22: Left: nonconvex 4-universal case $C 2$ (in bold lines). Right: folding a 4-link unit ruler $p_{0} p_{1} p_{2} p_{3} p_{4}$ into $C 2$.

prove that for $x=\sqrt{2-\sqrt{3}}=0.517 \ldots$, i.e., $c$ is at the middle point of arc $o b$, the nonconvex case $C 2$ shown in Figure 22 has the smallest area $0.295 \ldots$ in this family. In Section 4.3, we show that $C 2$ is a 4 -universal but not 5 -universal case. Thus, the upper bound of $B_{4}$ is improved from 0.486 to 0.296 . 


\subsection{Area of $S 2$}

In preparation for calculating the area of $C 2$, we provide the missing details in [1] for calculating the minimum area of $S 2$. In Section 4.2, the area of $C 2$ is derived based on the following calculations. The area of $S 2$ is the sum of the areas of its three parts: the isosceles triangles $\Delta b c d, \Delta a b d$ and the circular segment $c b$. In $\Delta b c d, \alpha=\arccos \frac{1}{2 x}$ and its area is $\frac{\sqrt{4 x^{2}-1}}{4}$. In isosceles triangle $\Delta a b c, \beta=\arccos \frac{x}{2}$ and its area is $\frac{x}{4} \sqrt{4-x^{2}}$. The area of the circular segment $c b$ is the area of the circular sector $a b c$ minus the area of $\Delta a b c$, i.e., $\frac{\pi}{2}-\arccos \frac{x}{2}-\frac{x}{4} \sqrt{4-x^{2}}$. In $\Delta a b d$, $\angle a b d=\beta-\alpha$, its area is $\sin (\beta-\alpha) / 2=\sin \left(\arccos \frac{x}{2}-\arccos \frac{1}{2 x}\right) / 2$. In summary,

$$
\begin{aligned}
\operatorname{area}(S 2) & =\operatorname{area}(\Delta b c d)+\operatorname{area}(\Delta a b d)+\operatorname{area}(\operatorname{circular} \text { segment } c b) \\
& =\frac{\sqrt{4 x^{2}-1}}{4}+\sin \left(\arccos \frac{x}{2}-\arccos \frac{1}{2 x}\right) / 2 \\
& +\frac{\pi}{2}-\arccos \frac{x}{2}-\frac{x}{4} \sqrt{4-x^{2}} .
\end{aligned}
$$

Taking derivative yields,

$$
\begin{aligned}
\frac{d(\operatorname{area}(S 2))}{d x} & =\frac{4+x^{2}}{4 \sqrt{4-x^{2}}}-\frac{\sqrt{4-x^{2}}}{4}+\frac{x}{\sqrt{4 x^{2}-1}} \\
& -\left(\frac{1}{2 x \sqrt{4 x^{2}-1}}+\frac{1}{2 \sqrt{4-x^{2}}}\right) \cos \left(\arccos \frac{x}{2}-\arccos \frac{1}{2 x}\right)
\end{aligned}
$$
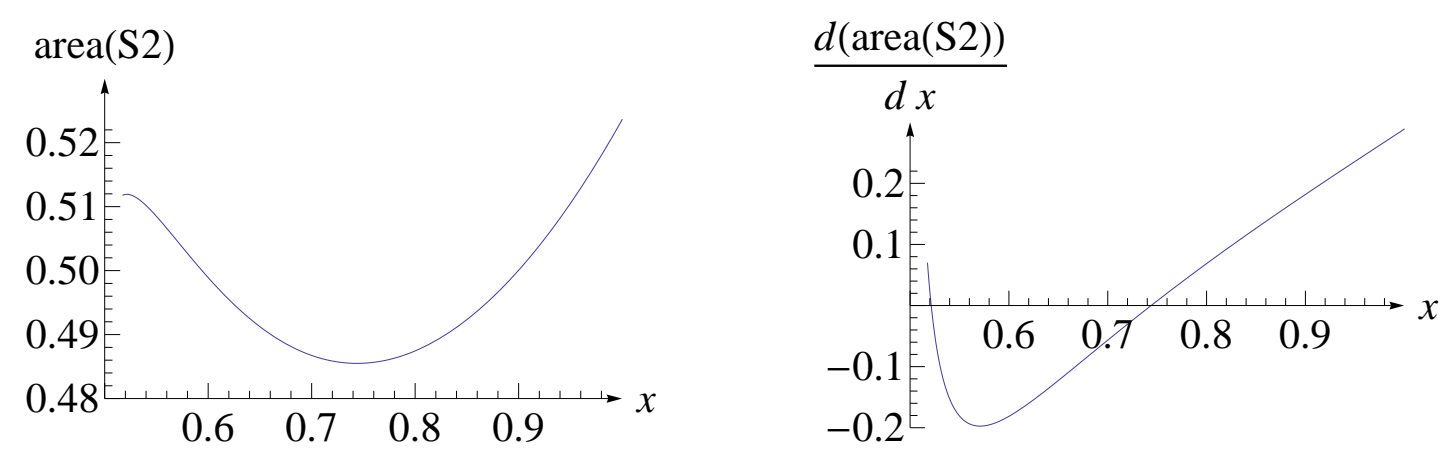

Figure 23: A plot of area(S2) (left) and its derivative (right). 
Solving for $\frac{d(\operatorname{area}(S 2))}{d x}=0$ yields two roots in $[0.5,1]$ (see Figure 23). When $x=0.743 \ldots, S 2$ has the smallest area area $(S 2) \leq 0.486$.

\subsection{Area of $C 2$}
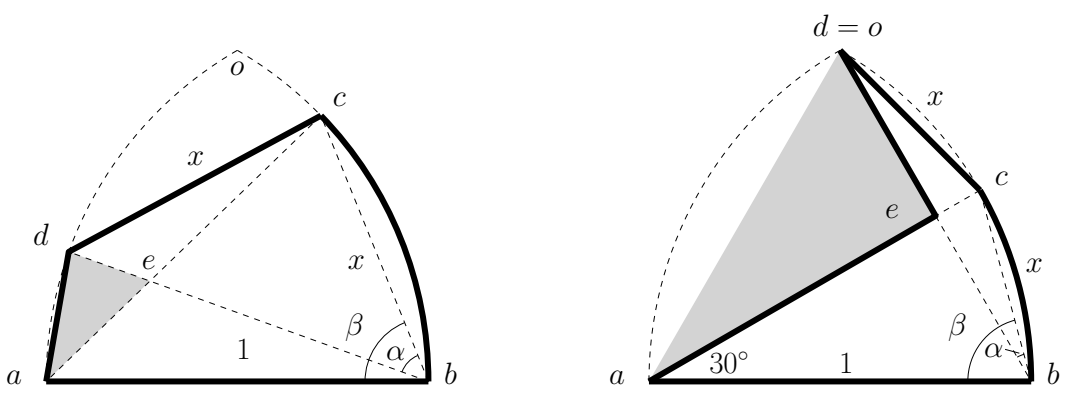

Figure 24: Derive $C 2$ from $S 2$. Left: $S 2$ (in bold lines) with the shaded triangle discarded. Right: $C 2$ (in bold lines) has minimum area $0.295 \ldots$ when $c$ lies in the middle of the circular arc $b d$.

Due to the subtraction of triangle $\Delta a d e$, we need to calculate the area of triangle $\Delta a b e$ instead of $\Delta a b d$ which is used in the area formula of $S 2$. In $\Delta a b c$ (see Figure 24 (left)), $\angle b a c=\pi-2 \beta$. In $\triangle a b e, \angle a b e=\beta-\alpha$ and

$$
\angle a e b=\pi-\angle b a e-\angle a b e=\pi-(\pi-2 \beta)-(\beta-\alpha)=\beta+\alpha .
$$

So $\frac{|b e|}{\sin (\pi-2 \beta)}=\frac{|a b|}{\sin (\beta+\alpha)}=\frac{1}{\sin (\beta+\alpha)}$, or $|b e|=\frac{\sin (2 \beta)}{\sin (\beta+\alpha)}$.

The area of $\Delta a b e$ is

$$
\begin{aligned}
\operatorname{area}(\Delta a b e) & =|a b| \cdot|b e| \sin (\beta-\alpha) / 2 \\
& =\frac{\sin \left(2 \arccos \frac{x}{2}\right) \sin \left(\arccos \frac{x}{2}-\arccos \frac{1}{2 x}\right)}{\sin \left(\arccos \frac{x}{2}+\arccos \frac{1}{2 x}\right)}
\end{aligned}
$$


Notice that

$$
\begin{aligned}
\sin \left(2 \arccos \frac{x}{2}\right) & =\frac{x \sqrt{4-x^{2}}}{2}, \\
\sin \left(\arccos \frac{x}{2}-\arccos \frac{1}{2 x}\right) & =\frac{\sqrt{4-x^{2}}-x \sqrt{4 x^{2}-1}}{4 x} \\
\sin \left(\arccos \frac{x}{2}+\arccos \frac{1}{2 x}\right) & =\frac{\sqrt{4-x^{2}}+x \sqrt{4 x^{2}-1}}{4 x} .
\end{aligned}
$$

Thus, the area of the convex case $C 2$ is

$$
\begin{aligned}
\operatorname{area}(C 2) & =\operatorname{area}(\Delta b c d)+\operatorname{area}(\operatorname{circular} \text { segment } c b)+\operatorname{area}(\Delta a b e) \\
& =\frac{\sqrt{4 x^{2}-1}}{4}+\frac{\pi}{2}-\arccos \frac{x}{2}-\frac{x}{4} \sqrt{4-x^{2}} \\
& +\frac{x\left(4-x^{2}\right)-x^{2} \sqrt{\left(4-x^{2}\right)\left(4 x^{2}-1\right)}}{4 \sqrt{4-x^{2}}+4 x \sqrt{4 x^{2}-1}}
\end{aligned}
$$

Taking derivative yields

$$
\begin{aligned}
\frac{d(\operatorname{area}(C 2))}{d x} & =\frac{4+x^{2}}{4 \sqrt{4-x^{2}}}-\frac{\sqrt{4-x^{2}}}{4}+\frac{x}{\sqrt{4 x^{2}-1}} \\
& -\frac{4 x\left(8 x^{2}-1\right)\left(4-x^{2}\right)}{\left(4 \sqrt{4-x^{2}}+4 x \sqrt{4 x^{2}-1}\right)^{2} \sqrt{4 x^{2}-1}} \\
& -\frac{4 x^{3} \sqrt{4 x^{2}-1}-32 x^{4} \sqrt{4-x^{2}}}{\left(4 \sqrt{4-x^{2}}+4 x \sqrt{4 x^{2}-1}\right)^{2}} \\
& +\frac{4-3 x^{2}-2 x \sqrt{\left(4-x^{2}\right)\left(4 x^{2}-1\right)}}{4 \sqrt{4-x^{2}}+4 x \sqrt{4 x^{2}-1}} \\
& -\frac{x^{2}\left(8 x\left(4-x^{2}\right)-2 x\left(4 x^{2}-1\right)\right)}{8\left(4-x^{2}\right) \sqrt{4 x^{2}-1}+8 x\left(4 x^{2}-1\right) \sqrt{4-x^{2}}} .
\end{aligned}
$$

We verify that $\frac{d(\operatorname{area}(C 2))}{d x}=0$ has no real root in $[\sqrt{2-\sqrt{3}}, 1]$. Indeed, as shown in Figure 25, area $(C 2)$ is a monotonically increasing function with respect to $x$ in this range. Thus, the area of $C 2$ is minimized when $x=\sqrt{2-\sqrt{3}}$, as depicted in Figure 24 (right). Since $c$ lies in the middle of the circular arc $b d$, line segment $c e$ 

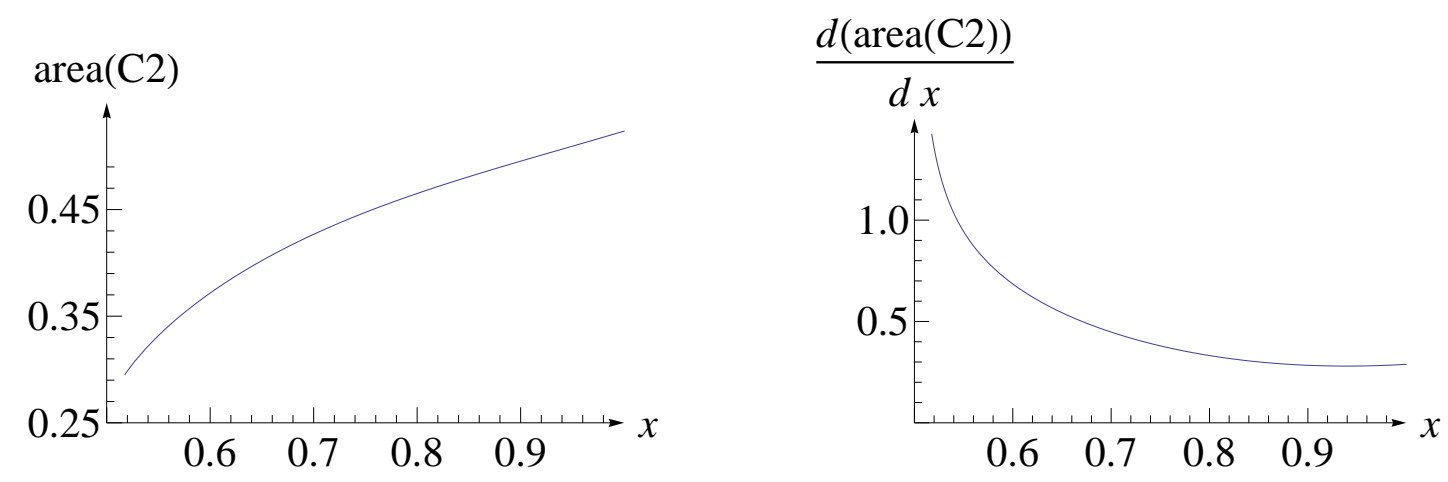

Figure 25: A plot of area $(C 2)$ (left) and its derivative (right).

is perpendicular to $e d$ and $|e d|=1 / 2,|c e|=\sqrt{7-4 \sqrt{3}} / 2$. The minimum area of $C 2$ is area $($ sector $a b c)+\operatorname{area}(\Delta c d e)=\pi / 12+\sqrt{7-4 \sqrt{3}} / 8=0.295 \ldots$

\section{3 $C 2$ is 4-universal}

First we show that any 3 -link unit ruler $p_{0} p_{1} p_{2} p_{3}$ can be folded into $C 2$ (see Figure 26).
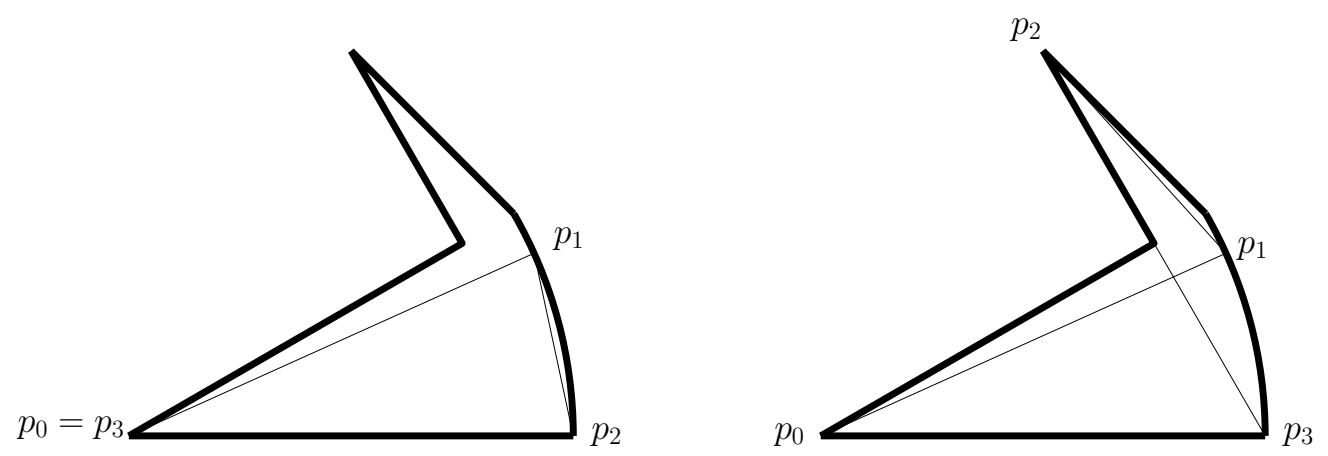

Figure 26: Two cases of folding a 3-link ruler into $C 2$ (in bold lines). Left: the middle link is short. Right: the middle link is long.

Lemma 3. C2 is a nonconvex 3-universal case.

Proof. It is sufficient to consider rulers with links $1, t, 1$ for any $t \in(0,1]$.

Case 1: $t$ is small, i.e., $t \leq x=\sqrt{2-\sqrt{3}}$. So both end points of the center link $t$ can be placed on the circular arc $b c$ (see Figure 27 (left)). Since arc $b c$ is 

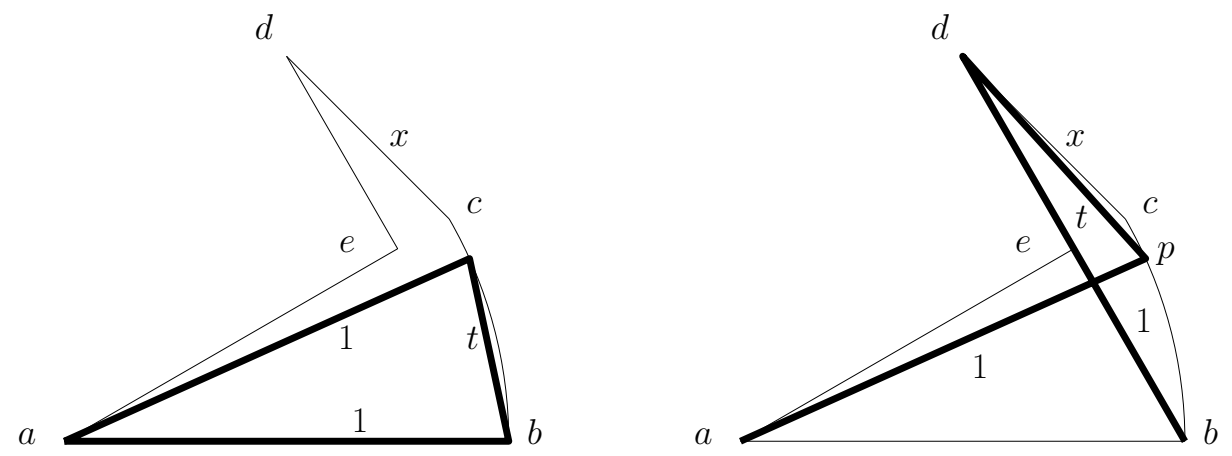

Figure 27: Fold a 3-link ruler (in bold lines) into $C 2$. Left: $t \leq x$. Right: $t>x$.

centered at $a$ with radius 1 , both links of length one can be folded from arc $b c$ to $a$.

Case 2: $t$ is large, i.e., $|c d|=x<t \leq 1=|b d|$. As shown in Figure 27 (right), starting from point $d$, there exists a point $p$ on $\operatorname{arc} b c$ such that $|d p|=t$. So the $1, t, 1$ ruler can be placed from $b$ to $d$ to $p$ to $a$.

Now we prove that any 4 -link unit ruler $p_{0} p_{1} p_{2} p_{3} p_{4}$ can be folded inside $C 2$ (as illustrated in Figure 28).
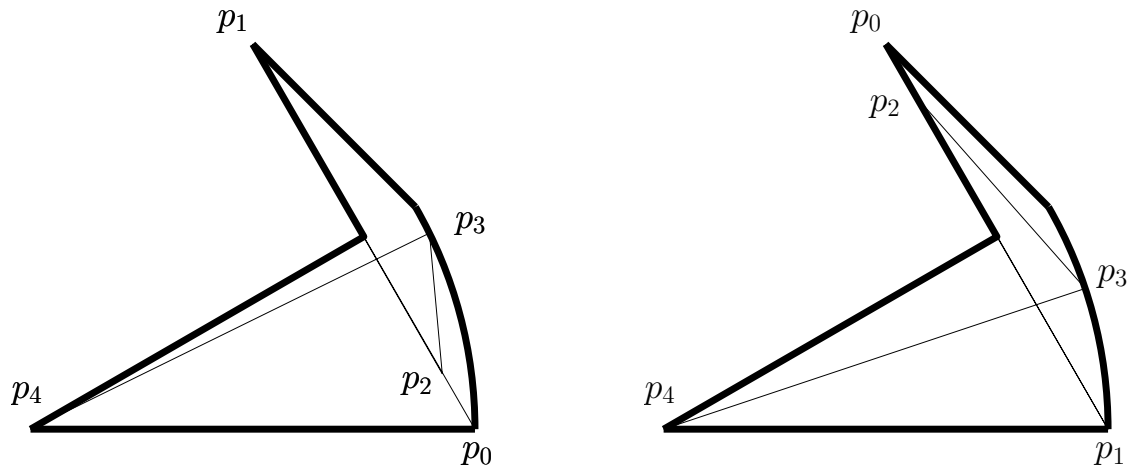

Figure 28: Two cases of folding a 4-link ruler into $C 2$ (in bold lines). Left: $p_{2} p_{3}$ is short. Right: $p_{2} p_{3}$ is long.

Lemma 4. $C 2$ is a nonconvex 4-universal case.

Proof. It is sufficient to consider unit rulers with links $1, t, t^{\prime}, 1$.

Case 1: $t+t^{\prime} \leq 1$. The folding problem of this ruler can be reduced to a folding problem of a 3 -link ruler $1, t+t^{\prime}, 1$ which is already solved by Lemma 3 . 
Case 2: $t+t^{\prime}>1$. Without loss of generality, we assume $t \geq t^{\prime}$. Label the end points of the ruler by $p_{i}, i=0,1,2,3,4$, such that $\left|p_{0} p_{1}\right|=\left|p_{3} p_{4}\right|=1,\left|p_{1} p_{2}\right|=t$ and $\left|p_{2} p_{3}\right|=t^{\prime}$. Fold the ruler such that the first two links overlap each other, i.e., $p_{2}$ lies on $p_{0} p_{1}$. As illustrated in Figure 29 (left), we place $p_{0} p_{1}$ at $b d$, then $p_{2}$ is on $e b$ otherwise $t+t^{\prime} \leq 1 .\left|p_{2} d\right|=t \geq t^{\prime}$ and $\left|p_{2} b\right|=1-t<t^{\prime}$, so there exists a point $p$ on the circular arc $b c d$ (notice that the arc $c d$ is imaginary) such that $\left|p_{2} p\right|=t^{\prime}$.

Case 2a: $p$ lies on arc $b c$. We can place $p_{3}$ at $p$ then $p_{4}$ at $a$ as illustrated in Figure 29 (left).

Case 2b: $p$ lies on (the imaginary) arc $c d$. We flip the ruler around with respect to the axis $a c$. As shown in Figure 29 (right), now $p_{0}$ is at $d, p_{1}$ is at $b$ and $p_{2}$ is at $p_{2}^{\prime}$. Point $p$ is also flipped to point $p^{\prime}$ on $\operatorname{arc} b c$. So we can place $p_{3}$ at $p^{\prime}, p_{4}$ at $a$.

Thus, in both cases the 4-link ruler can be folded into $C 2$. More formally, if $p_{0} p_{1}\left(\right.$ or $\left.p_{1} p_{0}\right)$ is placed at $b d$ and $p_{2}$ is folded on $p_{0} p_{1}$, the distance $\left|p_{2} c\right|=$ $\sqrt{(1 / 2-t)^{2}+(7 / 4-\sqrt{3})}$. If $\left|p_{2} c\right| \geq t^{\prime}$ (Case $\left.2 a\right), p_{0}$ is placed at $b$. Otherwise $\left|p_{2} c\right|<t^{\prime}$ (Case 2b), $p_{0}$ is placed at $d$. Then in both cases, there exists a point $p$ on arc $b c$ such that $\left|p_{2} p\right|=t^{\prime}$.
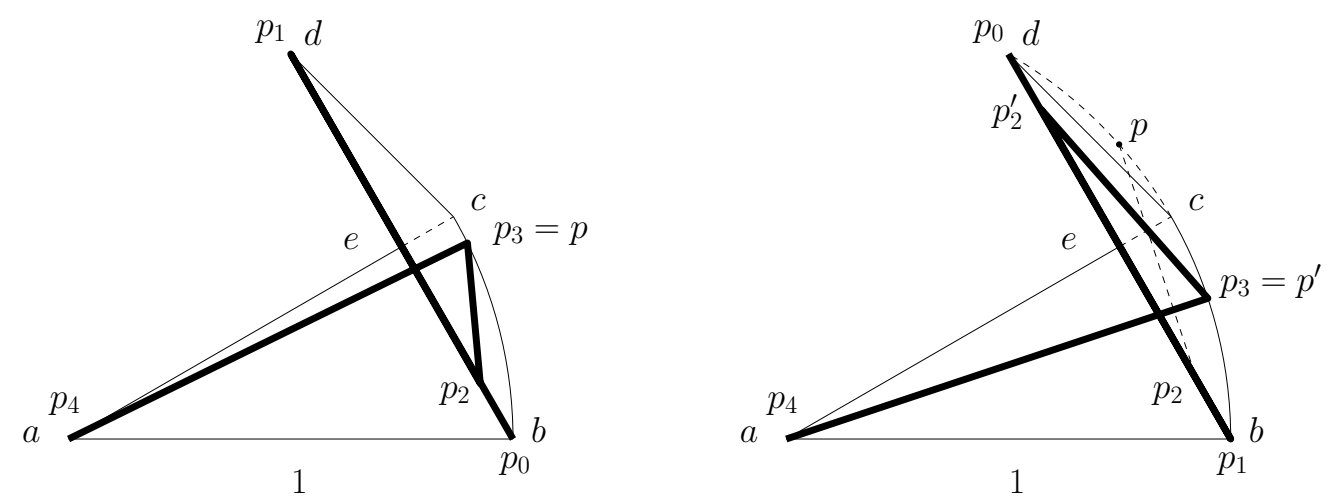

Figure 29: Fold a 4-link ruler (in bold lines) into $C 2$. Left: $\left|p_{2} c\right| \leq x$. Right: $\left|p_{2} c\right|>x$.

Hence Theorem 2 follows. Additionally we show that there exists a ruler with 5 links that cannot be folded inside $C 2$.

Lemma 5. C2 is not a 5-universal case. 
Proof. Consider folding the 5 -link ruler $1,0.6,1,0.6,1$ into $C 2$. The 0.6 links are in between the 1 links so both their end points must lie on $a, d$ or arc bc. But

since $1>0.6>\sqrt{2-\sqrt{3}}$, between $d$ and some point on arc $b c$ is the only possible position among all the combinations. Suppose the first 0.6 link is placed in this position, the other end points of the two 1 links adjacent to it must be placed at $b$ and $a$ respectively. But the second 0.6 link must also be placed to this only position which is impossible.

\section{$5 \quad$ Summary and Future Directions}

For the problem of finding minimum nonconvex universal cases for carpenter's rulers, we have shown:

- The area of a smallest nonconvex universal case with unit diameter is at most 0.583 .

- The area of any nonconvex universal case is at least 0.073 .

- The area of a smallest nonconvex 3-universal case is at most 0.296 and at least 0.038 .

- The area of a smallest nonconvex 4-universal case is at most 0.296.

In Section 3, the best possible lower bound given by one 3-link ruler is achieved, whereas the one given by a 5 -link ruler is not. Computer experiments suggest that 5-link rulers require folding area at least 0.137 ; more precisely:

- The minimum folding of a 5-link ruler with lengths 1, 0.6, 1, 0.6, 1 has (nonconvex) area at least 0.092 .

- The minimum folding of a 5 -link (symmetric) ruler with lengths $1, t, 1, t, 1$ has area at least 0.115 when $t=0.8$. 
- The minimum folding of a 5-link (asymmetric) ruler with lengths $1, t_{1}, 1, t_{2}, 1$ has area at least 0.137 when $t_{1}=0.7, t_{2}=0.4$.

The difficulty of approaching these better bounds lies in the complicated computations of nonconvex areas in many sub-cases. Note however that even the computational results were used, the resulting lower bounds would still be far away from the current upper bound of 0.583 , which we believe is closer to the truth.

Based on these observations, possible future research directions are:

1. Derive better lower bounds for universal cases using rulers with more links.

2. Derive better lower bounds for universal cases using combinations of multiple rulers.

Recall that the area of $R 1$ is $0.523 \ldots$ which is the current best upper bound for $A_{5}, A_{6}$ and $B_{5}, B_{6}$. In Section 4 , we improved the upper bound for $B_{4}$ (and $\left.B_{3}\right)$ to 0.296 with the nonconvex 4-universal case $C 2$. But we also showed that $C 2$ is not a 5 -universal case. Based on these results, following questions can be asked:

3. Is there a 3 -universal case with area smaller than the area of $C 2$, namely $0.296 ?$

4. Is there a 5 -universal case with area smaller than the area of $R 1$, namely $0.523 ?$

5. What are the tight bounds for $A_{k}$ and $B_{k}$ when $k=3,4,5$ and 6 ?

6. Are there convex $k$-universal cases for $k>6$ with area smaller than 0.614 , the area of the current best convex universal case $R 2$ ?

7. Are there $k$-universal cases for $k>6$ with area smaller than 0.583 , the area of the current best (nonconvex) universal case $C$ ?

8. Especially, we are interested in the connection between the problem of finding minimum area $k$-universal cases and the problem of finding minimum area 
universal cases. More specifically, is there a $k$ such that $A_{k}\left(B_{k}\right)$ matches the universal convex (nonconvex) bound?

Additionally, in [9], Klein and Lenz showed that no subset of $R 2$ with smaller area than $R 2$ is a universal case. This is proved by using a ruler with $n$ links where $n$ goes to infinity. The authors showed that the only possible folding inside $R 2$ covers the whole area of $R 2$. Motivated by this result, we propose the following interesting open problems:

9. Does a similar result hold for the nonconvex universal case $C$ ?

10. Does a similar result hold for the nonconvex 4-universal case $C 2$ ? Notice that in this problem, the method with rulers having the number of links going to infinity (which was used in [9]) is not applicable. Rulers are restricted to have at most 4 links. However, a possible approach is to use a combination of multiple rulers and show that no matter how the rulers are folded, $C 2$ will be covered. 


\section{References}

[1] H. Alt, K. Buchin, O. Cheong, F. Hurtado, C. Knauer, A. Schulz, and S. Whitesides, Small boxes for carpenter's rules, manuscript, 2006.

http://page.mi.fu-berlin.de/alt/papers/carpenter.pdf

[2] P. Braß, W. Moser, and J. Pach. Research Problems in Discrete Geometry, Springer, New York, 2005.

[3] P. Braß, M. Sharifi, A lower bound for Lebesgue's universal cover problem, International Journal of Computational Geometry and Applications, 15(5), 2005, pp. $537-544$.

[4] G. Călinescu and A. Dumitrescu, The carpenter's ruler folding problem, in J. Goodman, J. Pach and E. Welzl (editors), Combinatorial and Computational Geometry, Mathematical Science Research Institute Publications, Cambridge University Press, 2005, pp. 155-166.

[5] H. G. Eggleston, Minimal universal covers in $E^{n}$, Israel Journal of Mathematics, 1(3), 1963, pp. 149-155.

[6] J. Gerriets and G. Poole, Convex regions which cover arcs of constant length, The American Mathematical Monthly, 81, 1974, pp. 36-41.

[7] H. C. Hansen, The worm problem (in Danish), Normat, 40, 1992, pp. 119-123.

[8] J. E. Hopcroft, D. Joseph and S. Whitesides, Movement Problems for 2Dimensional Linkages. SIAM Journal on Computing, 13(3), 1984, pp. 610-629.

[9] O. Klein and T. Lenz, Carpenters rule packings - a lower bound, Abstracts of 23rd European Workshop on Computational Geometry, 2007, pp. 34-37.

[10] R. Norwood and G. Poole, An Improved Upper Bound for Leo Moser's Worm Problem. Discrete and Computational Geometry, 29(3), 2003, pp. 409-417.

[11] R. Norwood, G. Poole and M. Laidacker, The worm problem of Leo Moser, Discrete and Computational Geometry, 7(1), 1992, pp. 153-162.

[12] J. Pál, Über ein elementares Variationsproblem, Math.-fys. Medd., Danske Vid. Selsk., 3(2), 1920, pp. 2-35.

[13] J. Pál, Ein Minimumproblem für Ovale, Math. Annalen, 83, 1921, pp. 311319.

[14] G. Poole and J. Gerriets, Minimum covers for arcs of constant length. Bulletin of the American Mathematical Society, 79(2), 1973, pp. 462-463.

[15] J.E. Wetzel, Sectorial Covers for Curves of Constant Length. Canadian Math. Bull., 16, 1973, pp. 367-375. 